\title{
An Optimal Bound for High-Quality Conforming Triangulations*
}

\author{
Tiow-Seng Tan \\ Department of Information Systems and Computer Science, \\ National University of Singapore, Lower Kent Ridge Road, \\ Singapore 119260 \\ tants@iscs.nus.sg
}

\begin{abstract}
This paper shows that, for any plane geometric graph $\mathcal{G}$ with $n$ vertices, there is a triangulation $\mathcal{T}$ that conforms to $\mathcal{G}$, i.e., each edge of $\mathcal{G}$ is the union of some edges of $\mathcal{T}$, where $\mathcal{T}$ has $O\left(n^{2}\right)$ vertices with each angle of its triangles measuring no more than $\frac{11}{15} \pi$. Additionally, $\mathcal{T}$ can be computed in $O\left(n^{2} \log n\right)$ time.
\end{abstract}

\section{Introduction}

In many engineering applications such as finite-element analysis [7], surface interpolation [14], and shape reconstruction [6], two- and higher-dimensional domains are frequently decomposed into small and simple elements before numerical computation. One particularly important class of decompositions is the so-called simplicial cell complex, usually referred to as triangulation, where a domain is decomposed into simplicestriangles in two and tetrahedra in three dimensions-such that the intersection of two simplices is either empty or a face of both.

For a given domain such as a polygonal region or, more generally, a plane graph with straight edges, there are clearly many ways to decompose or triangulate it into a triangulation, with or without the addition of new vertices to the domain. However, accuracy and efficiency of an engineering computation impose optimal criteria such as the triangle shape (with bounds on triangle angles away from zero and $\pi$ ) and vertex size (with bounds on the number of new vertices), respectively. These criteria of shape and size are somewhat conflicting-good triangles may be the result of adding new vertices. Automatic generation of triangulations has been a subject for research since the 1960 s. Nevertheless, many interesting results, both practical and theoretical, have been

* This research was partially supported by the National University of Singapore under Grant RP940641. 
discovered in the recent years too; see, for example, [5], [8]-[10], [15]-[21], and the survey [4].

This paper considers triangulating a plane geometric graph, i.e., a plane graph with straight edges, using triangles with no large angles. Such resulting triangulations have potential applications in the area of finite-element and surface approximation; see, for example, [1], [2], and [12]. This paper shows that triangulating a plane geometric graph of $n$ vertices using angles no larger than $\frac{11}{15} \pi$ requires at most $\mathrm{O}\left(n^{2}\right)$ new vertices. The previous result by Mitchell [17] achieves angle bound of $\frac{7}{8} \pi$ with $O\left(n^{2} \log n\right)$ new vertices. This paper thus provides significantly better bounds on triangle shape and vertex size. The proof is constructive with relatively simple steps. The detailed argument about its correctness is however involved, requiring many new insights besides adapting some results from [17]. Note that the quadratic bound on the vertex size is within a constant factor of worst-case optimal; see [3] and [17] for discussions on the lowerbound construction due to M. S. Paterson.

The paper is organized as follows. Section 2 formalizes the problem. Section 3 provides the outline of the method that proves the quadratic bound, and Sections 4-9 discuss its details for angle bound $\alpha=\frac{3}{4} \pi$. Then Section 10 provides details on implementing the proof and extending it to $\alpha=\frac{11}{15} \pi$, and Section 11 concludes the paper. The Appendix documents some technical details required in Section 6.

\section{Some Terminologies}

We first introduce some notions, then define the problem.

Plane Geometric Graphs. Let $S$ be a set of $n$ points or vertices in $\mathfrak{\Re}^{2}$. An edge is a closed line segment connecting two points. Let $E$ be a collection of edges determined by vertices of $S$. Then $\mathcal{G}=(S, E)$ is a plane geometric graph if:

(i) No edge contains a vertex other than its endpoints, that is, $a b \cap S=\{a, b\}$ for every edge $a b \in E$.

(ii) No two edges cross, that is, $a b \cap c d \in\{a, b\}$ for every two edges $a b \neq c d$ in $E$.

One example of a plane geometric graph is a (simple) polygon where $E$ forms a single cycle. The cycle is the boundary of the polygon. It subdivides $\mathfrak{R}^{2}$ into a bounded face, its interior, and an unbounded face, its exterior. A polygon with four edges (or sides) is a quadrilateral.

Triangulations. A triangulation is a plane geometric graph $\mathcal{T}=(S, E)$ so that $E$ is maximal. By maximality, edges in $E$ bound the convex hull $c h(S)$ of $S$, i.e., the smallest convex set in $\mathfrak{H}^{2}$ that contains $S$, and subdivide its interior into disjoint faces bounded by triangles. With reference to a polygon, we talk about its triangulation as restricted to only its interior.

Conforming Triangulations. A plane geometric graph $\mathcal{G}^{\prime}=\left(S^{\prime}, E^{\prime}\right)$ conforms to another such graph $\mathcal{G}=(S, E)$ if $S \subseteq S^{\prime} \subset \operatorname{ch}(S)$ and each edge in $E$ is a union of 
edges in $E^{\prime}$. A triangulation $\mathcal{G}^{\prime}$ conforming to a plane geometric graph $\mathcal{G}$ is called a conforming triangulation of $\mathcal{G}$. Each vertex in $S^{\prime}-S$ is termed a Steiner vertex.

The problem studied here is as follows:

Given a plane geometric graph $\mathcal{G}=(S, E)$, find a conforming triangulation of $\mathcal{G}$ with a small vertex set and with each angle of its triangles measuring at most $\alpha$.

\section{The Outline of Construction}

Given a plane geometric graph $\mathcal{G}=(S, E)$ where $|S|=n$, the algorithm of Edelsbrunner et al. [11] can augment it by edges to a triangulation that minimizes its maximum angle over all possible augmentations. We are done if $\mathcal{T}$ has angles each measuring at most the targeted angle bound $\alpha$. Because of this and the fact that the size of any triangulation of $\mathcal{G}$ is a constant factor of the size of $\mathcal{G}$, we assume in our discussion that the given plane geometric graph $\mathcal{G}=(S, E)$ is actually a triangulation $\mathcal{T}$ and $\mathcal{T}$ has some bad angles. A bad angle is an angle with measure larger than $\alpha$, whereas a good angle is otherwise. For our discussion, the targeted angle bound $\alpha$ is set to $\frac{3}{4} \pi$ unless specified otherwise as in Section 10.

The main difficulty of the problem is as follows. Suppose $\mathcal{T}$ has a bad angle at $q$ in $\Delta p q r$. Then we can add a Steiner vertex, say $t$, on $p r$ to subdivide the bad angle at $q$ with the edge $t q$. This resolution however creates a "large angle" of $\pi$ at $t$ in the triangle, say $p r s$, sharing the edge $p r$ with $\Delta p q r$. In other words, the problem of a large angle has propagated to $\Delta p r s$ and another subdivision is necessary. Unless $t s$ can subdivide this large angle into two good angles or $\Delta p r s$ does not exist as $p r$ is actually an edge bounding $\operatorname{ch}(S)$, we need to add one Steiner vertex on either $p s$ or $r s$ and the situation continues to propagate each time to another adjacent triangle. The sequence of Steiner vertices generated in this manner can be viewed as forming a propagation path with edges joining two consecutive Steiner vertices. A successful approach to add a bounded number of Steiner vertices has to terminate each propagation path effectively. This is achieved in this paper by fences (Section 4) and dead-ends (Section 5).

The proposed construction has six major steps. It first subdivides each triangle of $\mathcal{T}$ into three quadrilaterals (Section 4) and works with these quadrilaterals throughout the construction. In the second and third steps (Sections 5 and 6) it decides where to add Steiner vertices forming propagation paths. To control the number of Steiner vertices, the construction bounds the number of vertices in each propagation path to linear in size. In the fourth and fifth steps (Sections 7 and 8) the construction manipulates edges of propagation paths to remove all crossings among edges of propagation paths. As a result, some Steiner vertices added by the third step may be removed. In the last step (Section 9) the construction triangulates each quadrilateral one by one using edges of propagation paths lying in the quadrilateral. The output is the union of triangulations of all quadrilaterals.

\section{Erecting Fences}

For $\Delta p q r$, let $c$ be the center of its inscribing circle. Each edge joining $c$ with the perpendicular projection of $c$ onto a side of $\Delta p q r$ is a spoke of $\Delta p q r$. For example, 


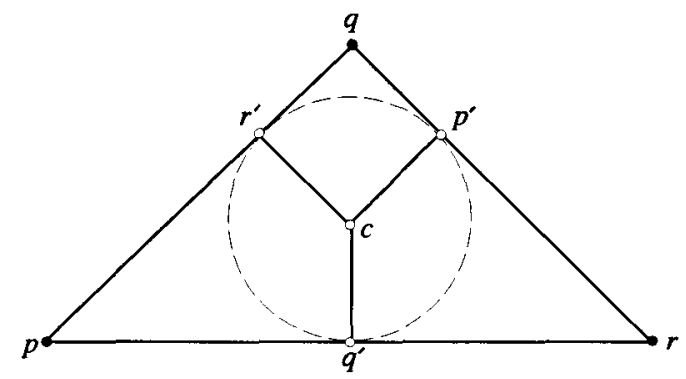

Fig. 4.1. Note that $\left|p r^{\prime}\right|=\left|p q^{\prime}\right|,\left|r p^{\prime}\right|=\left|r q^{\prime}\right|,\left|q r^{\prime}\right|=\left|q p^{\prime}\right|$, and $\left|c r^{\prime}\right|=\left|c p^{\prime}\right|=\left|c q^{\prime}\right|$.

in Fig. $4.1 c r^{\prime}, c q^{\prime}$, and $c p^{\prime}$ are the three spokes of $\Delta p q r$. For convenience, we let $\beta=\pi-\alpha$.

Step 1: Erecting Fences. For each $\triangle p q r \in \mathcal{T}$, add its spokes $c r^{\prime}, c p^{\prime}$, and $c q^{\prime}$ to subdivide it into three quadrilaterals $p q^{\prime} c r^{\prime}, r q^{\prime} c p^{\prime}$, and $q r^{\prime} c p^{\prime}$ as shown in Fig. 4.1. Next, mark $p q^{\prime} c r^{\prime}$ as a fence if $\angle p \geq \beta$, else as a nonfence. Similarly, mark $r q^{\prime} c p^{\prime}$ according to $\angle r$ and mark $q r^{\prime} c p^{\prime}$ according to $\angle q$.

The main purpose of fences is to stop each newly created Steiner vertex (introduced by later steps) lying on a boundary edge of some fence from further generating Steiner vertices. The next lemma shows that each fence with or without Steiner vertices on its boundary edges can always be triangulated with only good triangles. A good triangle is a triangle with no bad angle. For convenience, we treat each edge bounding $\operatorname{ch}(S)$ as a (degenerate) fence since fences and edges of $\operatorname{ch}(S)$ both terminate the generation of more Steiner vertices.

Lemma 4.1. Let $p q^{\prime} c r^{\prime}$ be a fence with $\angle q^{\prime}=\angle r^{\prime}=\pi / 2$ and $\angle p \geq \beta$. Then the region bounded by $p q^{\prime} c r^{\prime}$, possibly with other vertices on $p r^{\prime}$ and $p q^{\prime}$, can always be triangulated with only good triangles.

Proof. When there are no other vertices on $p r^{\prime}$ and $p q^{\prime}$, we are done as $p q^{\prime} c r^{\prime}$ can be triangulated with two right-angle triangles $p q^{\prime} c$ and $p r^{\prime} c$. Now, suppose there are other vertices along $p r^{\prime}$ and $p q^{\prime}$. Let the vertices along $p r^{\prime}$ be sorted as $p=r_{0}, r_{1}, \ldots, r_{m}=r^{\prime}$ with increasing distance from $p$. Similarly, let $p=q_{0}, q_{1}, \ldots, q_{l}=q^{\prime}$ be the corresponding sequence along $p q^{\prime}$. We now triangulate $p q^{\prime} c r^{\prime}$ to prove the claim. There are two cases: $2 \beta \leq \angle p<\pi$ and $\beta \leq \angle p<2 \beta$. For the first case, we simply add edges $c q_{i}$, for $i=0$ to $l-1$, and edges $c r_{j}$, for $j=1$ to $m-1$. Each triangle obtained is of the form $c q_{i} q_{i+1}$ or $c r_{j} r_{j+1}$. For the former, we have $\angle c q_{i} q_{i+1}<\pi / 2$ and $\angle q_{i} c q_{i+1}<\pi / 2$ because $\Delta c q_{i} q^{\prime}$ has a right angle at $q^{\prime}$. Also, $\angle c q_{i+1} q_{i}<\pi-\beta=\alpha$ because in $\Delta c q_{0} q_{i+1}$ we have $\angle q_{0}=\frac{1}{2} \angle r^{\prime} p q^{\prime} \geq \beta$. So $\Delta c q_{i} q_{i+1}$ is good. Similarly, we have a good $\Delta c r_{j} r_{j+1}$.

We next consider the case where $\beta \leq \angle p<2 \beta$. Initially, set $i=1, j=1$. Add the edge $r_{j} q_{i}$, and then increment $j$ if $r_{j}$ is closer to $p$ than $q_{i}$, else increment $i$. Repeat the previous statement until $p q^{\prime} c r^{\prime}$ is triangulated. Triangle $r_{m} q_{l} c$, the last one obtained, has 
clearly good $\angle r_{m}$ and $\angle q_{l}$, and also good $\angle c$ because $\angle c=\pi-\angle p \leq \pi-\beta=\alpha$. Triangle $p r_{1} q_{1}$, the first one obtained, is good because $\beta \leq p<2 \beta<\alpha$. The rest of the triangles are either of the form $r_{j-1} r_{j} q_{i}$ or $q_{i-1} q_{i} r_{j}$. We just consider $\Delta r_{j-1} r_{j} q_{i}$; similar argument applies to $\Delta q_{i-1} q_{i} r_{j}$. By construction, we have $r_{j-1}$ closer to $p$ than $q_{i}$, i.e., $\angle r_{j-1}>\angle q_{i}$ in $\triangle p r_{j-1} q_{i}$. Since $\angle p<2 \beta$, we have $\angle q_{i} r_{j-1} p>(\pi-2 \beta) / 2$. Hence, $\triangle r_{j-1} r_{j} q_{i}$ has $\angle r_{j-1}=\pi-\angle q_{i} r_{j-1} p<\pi / 2+\beta \leq \alpha$. Its $\angle r_{j}$ and $\angle q_{i}$ are also good because $\angle r_{j} p q_{i} \geq \beta$. So, it is a good triangle and the proof is complete.

\section{Setting Traps}

The second step, consisting of planning and adding substeps, is to augment $\mathcal{T}$ with a linear number of Steiner vertices, termed dead-ends. We need some terminologies for our discussion.

Paths. A path with origin $p_{0}$ and last vertex $p_{k}$ is a sequence of vertices $p_{0}, p_{1}, p_{2}$, $\ldots, p_{k}$ where each vertex lies on some edge of $\mathcal{T}$ and each line segment joining consecutive vertices $p_{i}$ and $p_{i+1}$ does not cross any edge of quadrilaterals. The line segment joining $p_{i}$ and $p_{i+1}$ is an $e d g e$ of the path or, more specifically, a directed edge $\overrightarrow{p_{i} p_{i+1}}$ with tail $p_{i}$ and head $p_{i+1}$. For $\overrightarrow{p_{i} p_{i+1}}$ lying in $p q^{\prime} c r^{\prime}$ with $p_{i} \in p q^{\prime}$ and $p_{i+1} \in p r^{\prime}$, we refer to $\angle p_{i} p_{i+1} p$ and $\angle p_{i} p_{i+1} r^{\prime}$ as the two angles at its head, and $\angle p_{i+1} p_{i} p$ and $\angle p_{i+1} p_{i} q^{\prime}$ as the two angles at its tail. Formally, each propagation path is a path where the two angles at the tail of each of its directed edges are good.

Path $P$ with vertices $p_{0}, p_{1}, p_{2}, \ldots, p_{k}$ and path $Q$ with vertices $q_{0}, q_{1}, q_{2}, \ldots, q_{k}$ are said to be coherent if $p_{i} p_{i+1}$ and $q_{i} q_{i+1}$, for each $0 \leq i \leq k-1$, lie in the same quadrilateral. They are parallel if $p_{i} p_{i+1}$ and $q_{i} q_{i+1}$, for each $0 \leq i \leq k-1$, lying in the same quadrilateral are parallel. The length of a path is equal to its number of directed edges.

A backward path is a path where the two angles at the head of each directed edge are $\alpha$ and $\beta$, and all good angles of $\beta$ at the heads are on the same side along the path. Analogously, a forward path is a path where the two angles at the tail of each directed edge are $\alpha$ and $\beta$, and all good angles of $\beta$ at the tails are on the same side along the path.

Planning Dead-Ends. To plan for a sufficient number of dead-ends, Step 2a computes, for each point $x$ of each quadrilateral edge, whether a good propagation path exists, i.e., a propagation path of length $K$ with origin $x$ and last vertex at an endpoint of a spoke where $K<6 n$ is twice the number of edges of $\mathcal{T}$. From this, we can view each quadrilateral edge, with respect to its quadrilateral, as consisting of alternating good and (open) bad segments where the former includes points that have good propagation paths whereas the latter is otherwise. Some of these bad segments will be used by Step $2 b$ to add dead-ends.

Step 2a: Planning Dead-ends. Consider each nonfence $p q^{\prime} c r^{\prime}$; see Fig. 5.1. We work on $q^{\prime}$ and similarly on $r^{\prime}$. Trace a backward path starting at $q^{\prime}$ with its second vertex $q_{1}^{\prime \prime}$ on $p r^{\prime}$ such that $\angle q^{\prime} q_{1}^{\prime \prime} r^{\prime}=\beta$. We first mark $q_{1}^{\prime \prime} r^{\prime}$ as a (part of some) good segment with respect to $p q^{\prime} c r^{\prime}$. At each extension of the backward path from length $i$ at $q_{i}^{\prime \prime}$ to length $i+1$ at 


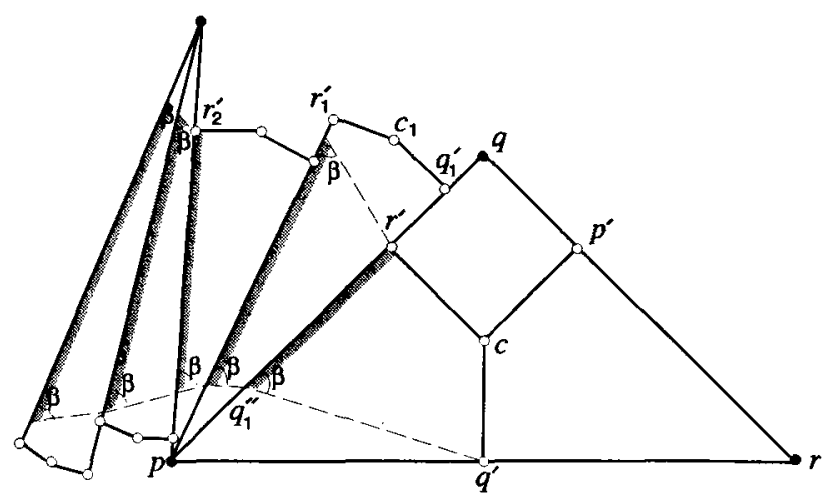

Fig. 5.1. The first five good segments identified due to the backward path with origin $q^{\prime}$ are shown shaded.

$q_{i+1}^{\prime \prime}$, where $q_{i}^{\prime \prime} \in p_{i} q_{i}^{\prime}$ and $q_{i+1}^{\prime \prime} \in p_{i} r_{i}^{\prime}$ of nonfence $p_{i} q_{i}^{\prime} c_{i} r_{i}^{\prime}$ (with $\angle q_{i}^{\prime}=\angle r_{i}^{\prime}=\pi / 2$ ), we mark $q_{i+1}^{\prime \prime} t_{i+1}^{\prime \prime}=q_{i+1}^{\prime \prime} s_{i+1}^{\prime \prime} \cap p_{i} r_{i}^{\prime}$ as a (part of some) good segment with respect to $p_{i} q_{i}^{\prime} c_{i} r_{i}^{\prime}$, where $s_{i+1}^{\prime \prime}$ is the point on the line through $p_{i} r_{i}^{\prime}$ with $\angle t_{i}^{\prime \prime} s_{i+1}^{\prime \prime} q_{i+1}^{\prime \prime}=\beta$ and $t_{i}^{\prime \prime}$ is an endpoint of the good segment $q_{i}^{\prime \prime} t_{i}^{\prime \prime}$ identified (with respect to $p_{i-1} q_{i-1}^{\prime} c_{i-1} r_{i-1}^{\prime}$ ) by the previous extension. The backward path terminates when one of the following conditions holds: it reaches an edge of a fence, or an edge for the third time, or it will cross a spoke in the next extension.

A few remarks are in order. First, we could have included in the definition of a good propagation path, one of bounded length to some edge of a fence. This is, however, not efficient for computation. Second, for the same reason, Step 2a identifies some but not all good segments, and simply takes the remaining as bad segments. Third, vertices of backward paths in Step 2a as well as forward paths in Step $2 \mathrm{~b}$ are for discussion purposes only and are not Steiner vertices added to $\mathcal{T}$; only dead-ends of traps are Steiner vertices added to $\mathcal{T}$ by Step $2 \mathrm{~b}$.

Adding Dead-Ends. A trap consists of a base $x y$, dead-ends $x^{\prime}$ and $y^{\prime}$, and boundary paths $P_{x}$ from $x$ to $x^{\prime}$ and $P_{y}$ from $y$ to $y^{\prime}$. Note that dead-ends $x^{\prime}, y^{\prime}$ may be the same point, and boundary paths $P_{x}$ and $P_{y}$, excluding their last edges, are coherent forward paths starting at $x$ and $y$ so that those good angles of $\beta$ at tails of their directed edges are inside the region bounded by $P_{x}, P_{y}, x y$, and $x^{\prime} y^{\prime}$.

Step 2b: Adding Dead-ends. Consider each nonfence $p q^{\prime} c r^{\prime} \subset p q r$; see Fig. 5.2(i). We discuss the following for $r^{\prime}$, and treat $q^{\prime}$ similarly. Let $p_{1} r_{1}^{\prime} c_{1} q_{1}^{\prime} \not \subset \Delta p q r$ be the nonfence incident to $p q$ (if $p q$ is not a convex hull edge) with $r^{\prime} \in p_{1} q_{1}^{\prime}$. If $r^{\prime}$ is an endpoint of a bad segment $x_{1} r^{\prime} \subseteq p_{1} q_{1}^{\prime}$ with respect to $p_{1} r_{1}^{\prime} c_{1} q_{1}^{\prime}$, we construct a trap with base $x_{1} r^{\prime}$ in the direction into $p_{1} r_{1}^{\prime} c_{1} q_{1}^{\prime}$. Next, let $p_{z} q_{z}^{\prime} c_{z} r_{z}^{\prime}$ be the nonfence containing the last edge of the backward path traced from $r^{\prime}$ at Step 2a. If the head of this last edge is an endpoint of some bad segment with respect to $p_{z} q_{z}^{\prime} c_{z} r_{z}^{\prime}$, we construct, in the direction into $p_{z} q_{z}^{\prime} c_{z} r_{z}^{\prime}$, a trap with the base at this bad segment; see Fig. 5.2(ii). 

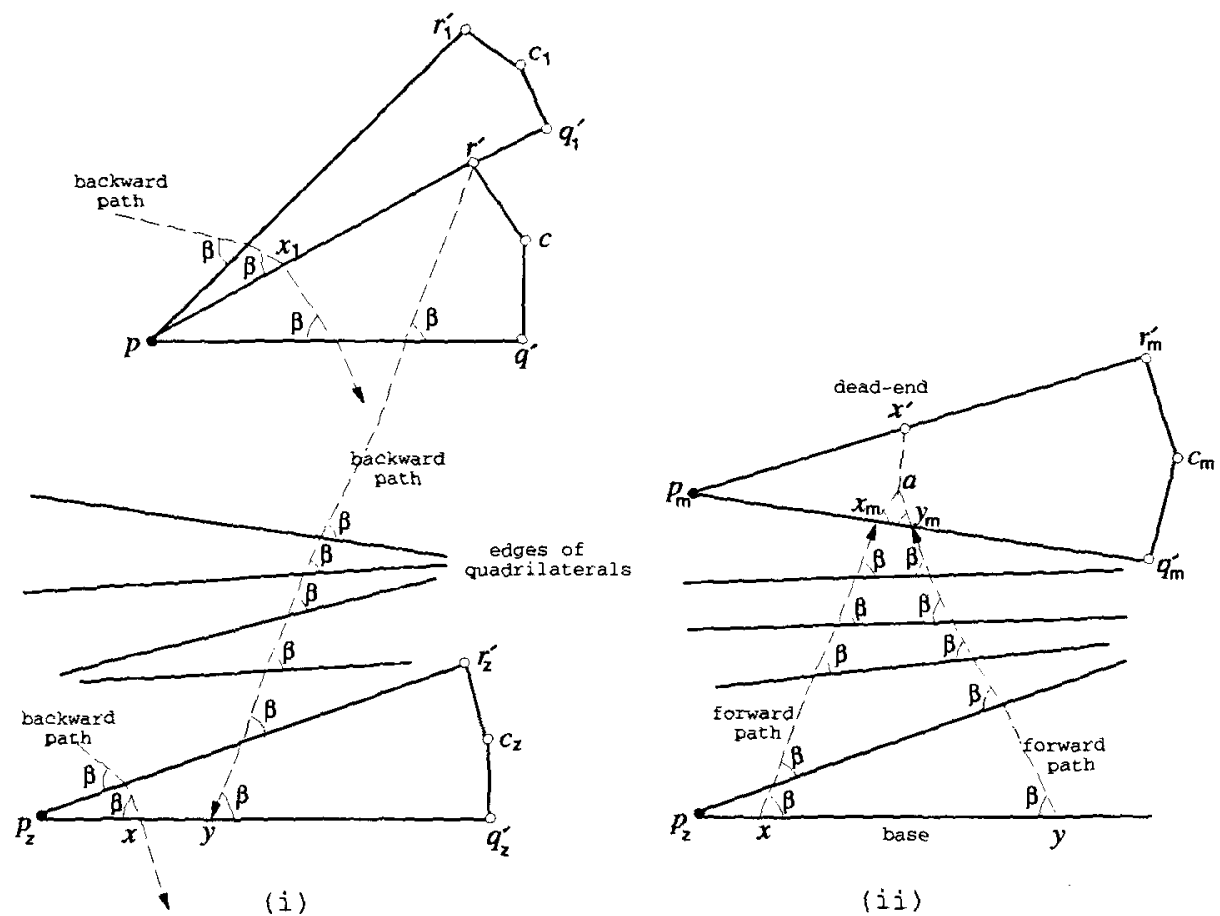

(ii)

Fig. 5.2. (i) The backward path traced from $r^{\prime}$ in Step 2a terminates at $y$. Segment $x y$ is a bad segment with respect to $p_{z} q_{z}^{\prime} c_{z} r_{z}^{\prime}$, so a trap with base $x y$ in the direction into $p_{z} q_{z}^{\prime} c_{z} r_{z}^{\prime}$ is constructed as shown in (ii).

To construct, in the direction into, say, $p_{z} q_{z}^{\prime} c_{z} r_{z}^{\prime}$, a trap with base $x y$ on $p_{z} q_{z}^{\prime}$ where $y \in x q_{z}^{\prime}$, we trace two coherent forward paths at $x$ and $y$ into $p_{z} q_{z}^{\prime} c_{z} r_{z}^{\prime}$ so that the paths move closer to each other as their lengths increase. Both paths continue until they have just reached, say, $\Delta p_{m} q_{m} r_{m}$ (with endpoints of spokes $p_{m}^{\prime} \in r_{m} q_{m}, r_{m}^{\prime} \in p_{m} q_{m}, q_{m}^{\prime} \in p_{m} r_{m}$ ), at, say, $x_{m} \in p_{m} q_{m}^{\prime}$ (possibly $x_{m}=x$ ) and $y_{m} \in x_{m} q_{m}^{\prime}$ (possibly $y_{m}=y$ ), where:

1. The two forward paths will intersect at a point, say $a$, in the interior of $p_{m} q_{m}^{\prime} c_{m} r_{m}^{\prime}$ when further extended for one more step (as in Fig. 5.2(ii)), or

2. $p_{m} q_{m}^{\prime}$ is the very first edge visited for the third time by both forward paths.

For case 1 , we consider $x^{\prime} \in p_{m} q_{m}$ where $a x^{\prime}$ is perpendicular to $x_{m} y_{m}$. We take $x^{\prime}$ as the dead-end if $a x^{\prime}$ does not intersect $c_{m} r_{m}^{\prime}$; otherwise, take $r_{m}^{\prime}$ as the dead-end. We complete the boundary paths of the trap by joining both forward paths to the dead-end. For case 2, $x_{m}$ and $y_{m}$ are the dead-ends and both forward paths are the boundary paths of the trap. The following result is immediate because of case 2 .

Lemma 5.1. The length of each boundary path of a trap is at most $K$.

In the above we ignore the fact that:

(i) $x$ may be the same as $p_{z}$ so a forward path from $p_{z}$ is not well defined. 
(ii) The two coherent forward paths traced may first hit an edge of a fence.

(iii) The two forward paths reach $\Delta p_{m} q_{m} r_{m}$ such that $x_{m} \in p_{m} q_{m}^{\prime}$ and $y_{m} \in q_{m}^{\prime} r_{m}$ where we can no longer maintain coherent forward paths in their extensions.

In all these we can actually ignore the construction of a trap. The reason for (i) is explained in Section 6 (in the proof of Lemma 6.3), for (ii) it is that each point on $x y$ after all has propagation paths of linear length to an edge of a fence, and for (iii) it is as follows. The point $x_{m}$ is so that $x_{m} \in r_{m}^{\prime \prime} q_{m}^{\prime} \subset p_{m} q_{m}^{\prime}$ of nonfence $p_{m} q_{m}^{\prime} c_{m} r_{m}^{\prime}$ where $\angle r_{m}^{\prime} r_{m}^{\prime \prime} q_{m}^{\prime}=\beta$, and a similar statement holds for $y_{m}$; thus, $x y$ is a good segment not identified by Step $2 \mathrm{a}$. This is so because the region $\mathcal{R}$ enclosed by $P_{x}, x_{m} y_{m}, P_{y}, y x$ (where $P_{x}$ and $P_{y}$ are the two forward paths from $x$ to $x_{m}$ and $y$ to $y_{m}$, respectively) is emptied of endpoints of spokes, except for $q_{m}^{\prime} \in x_{m} y_{m}$. So, if $r_{m}^{\prime \prime}$ is an interior point of $x_{m} q_{m}^{\prime}$, then the backward path from $r_{m}^{\prime}$ traced at Step 2a inside $\mathcal{R}$ is parallel to $P_{x}$ and reaches $x y$, and Step 2a should have identified some part of $x y$ as a good segment.

Lemma 5.2. The number of traps constructed in Step $2 \mathrm{~b}$ is at most $14 n$.

Proof. Consider $\triangle p q r \in \mathcal{T}$ with endpoints of spokes $p^{\prime} \in r q, r^{\prime} \in p q, q^{\prime} \in p r$ as in Fig. 5.1. It is easy to check that at least one of the three quadrilaterals, say $q r^{\prime} c p^{\prime}$, is a fence. So we trace at most one backward path each at $r^{\prime}$ and $p^{\prime}$, and at most two at $q^{\prime}$. These backward paths result in at most eight traps but at most seven is necessary since the two with bases at $q^{\prime}$, if any, can always be combined into one (and we pretend we did so in the construction). The claim follows as there are at most $2 n$ triangles.

\section{Generating Paths}

The necessity to propagate vertices in computing triangulation with angles bounded away from $\pi$ is a consequence of the following simple lemma introduced in [11].

Lemma 6.1. If $x y$ is an edge in a triangulation $\mathcal{A}$ of a point set $S$, then $\mu(\mathcal{A}) \geq$ $\max _{s \in S} \angle x$ sy where $\mu(\mathcal{A})$ denotes the largest angle in $\mathcal{A}$.

In effect, propagation is to subdivide each long edge into smaller ones so as to remove large angles subtended by the edge.

We adapt a few terminologies from [17]. Let $s$ be a point on $p q^{\prime}$ of nonfence $p q^{\prime} c r^{\prime}$ with $\angle q^{\prime}=\angle r^{\prime}=\pi / 2$. The cone at $s$ consists of all points $t$ of $p q^{\prime} c r^{\prime}$ such that both $\angle t s p$ and $\angle t s q^{\prime}$ are at most $\alpha$. The maw of $s$ is the portion of the cone at $s$ on the boundary of $p q^{\prime} c r^{\prime}$, except for $s$. Note that each point on the maw of $s$ is a candidate for extending a propagation path from $s$. Next, the iterative construction of a union of cones at points in maws, starting with $s$, results in the horn of $s$. Initially at stage 0 , the horn is the cone at $s$. The horn at stage $i+1$ is the union of cones at points in the union of maws, or simply maw, at stage $i$; see Fig. 6.1. It is clear that the two paths bounding the horn of $s$ are actually forward paths starting at $s$. They are referred as the boundary paths of the horn. 


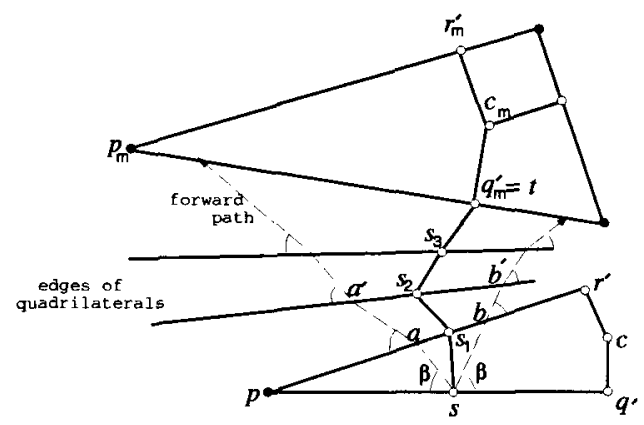

Fig. 6.1. The horn at stage 0 is the cone $s a b$, and the maw at this stage is $a b$. The horn at stage 1 is the region bounded by the two forward paths from $s$ to $a^{\prime}$ and $s$ to $b^{\prime}$, and the maw $a^{\prime} b^{\prime}$ at this stage. At stage 3 , the horn at $s$ contains $q_{m}^{\prime}$, and a propagation path such as $s, s_{1}, s_{2}, s_{3}, q_{m}^{\prime}$ can be constructed from $q_{m}^{\prime}$ back to $s_{3}$ (with $q_{m}^{\prime}$ in the maw of $s_{3}$ ), then $s_{3}$ to $s_{2}$ (with $s_{3}$ in the maw of $s_{2}$ ), and so on.

To generate a propagation path with origin $s$, the construction first computes iteratively the hom of $s$ until it intersects some point $t$ which can be an endpoint of a spoke (on some edge of $\mathcal{T}$ ), or a dead-end, or any point on an edge of a fence, or a vertex of some existing propagation path, then works straightforwardly from $t$ back to $s$. Besides the above, $t$ can possibly be some point common to cones of the horn from different stages, which happens when the horn of $s$ self-intersects; see Fig. 6.2. For all these, we need to place two restrictions on the above choices of $t$ so as to say that a propagation path terminates properly and to call it a properly terminating propagation path: $P$ can terminate at a dead-end only when the horn of $s$ has entered the corresponding trap through the base of the trap, and it can terminate at vertex $t$ of some propagation path $P^{\prime}$ (possibly $P$ itself) only when the last edge of $P$ and some edge of $P^{\prime}$ share $t$ as the head in the same nonfence; see Fig. 6.3. These are to avoid having a Steiner vertex (other than an endpoint of a spoke) to serve both as a head of some directed edge and a tail of another directed

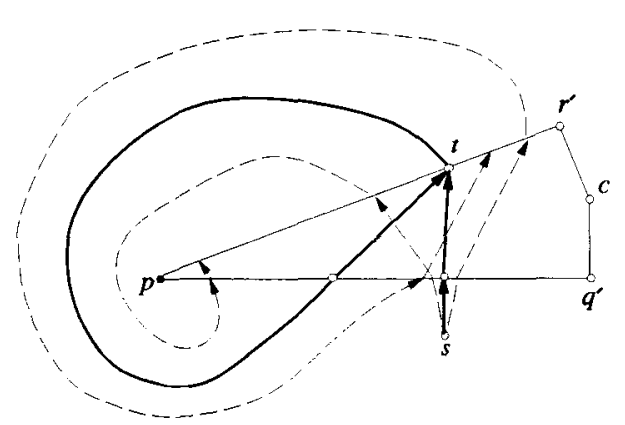

(i)

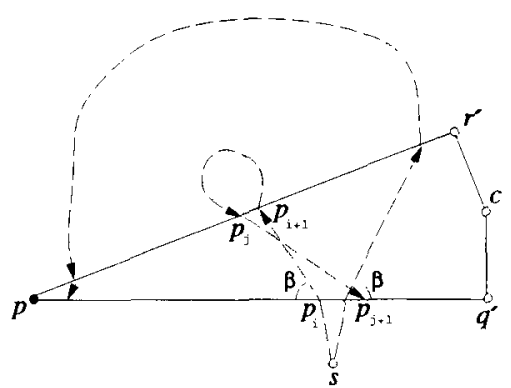

(ii)

Fig. 6.2. The horn self-intersects while passing through $p q^{\prime} c r^{\prime}$ in the same direction as in (i) or in the opposite directions as in (ii). The latter is not used to derive propagation paths, whereas the former is used when the intersection contains a vertex such as $t$, whose horn lying inside the horn of $s$ contains itself at some later stage. 


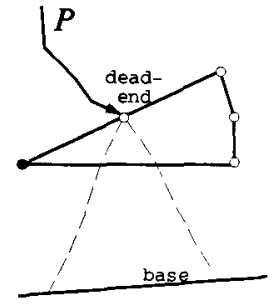

(i)

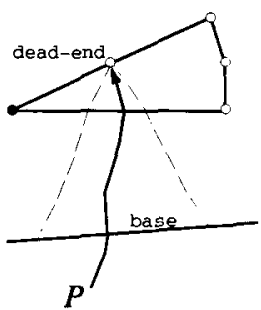

(ii)

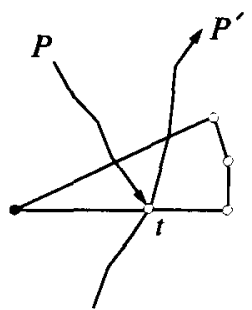

(iii)

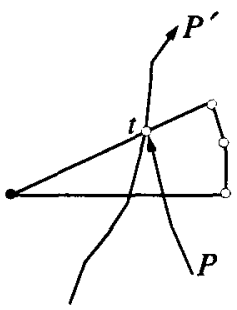

(iv)

Fig. 6.3. Propagation path $P$ terminates properly at a dead-end as in (ii) or at some vertex $t$ of $P^{\prime}$ as in (iv). The two restrictions prohibit the termination of $P$ as in (i) and (iii).

edge lying in the same nonfence. Also, the construction maintains that propagation paths do not cross spokes. This is possible because of the simple fact that whenever a horn intersects spokes $c q^{\prime}$ or $c r^{\prime}$ inside $p q^{\prime} c r^{\prime}$ upon entering from $p q^{\prime}$, it also contains $r^{\prime}$.

Step 3: Generating Paths. Consider endpoints of spokes that are on edges of $\mathcal{T}$ one by one. With each such endpoint as the first vertex, generate a (short) properly terminating propagation path of linear length. Then, for each dead-end that terminates some propagation paths constructed so far, generate a (short) properly terminating propagation path of linear length with this dead-end as the origin. At the end, those dead-ends that do not terminate any propagation paths are removed.

The next three lemmas imply the quadratic bound on the number of Steiner vertices. Two directed edges $\overrightarrow{a b}$ and $\overrightarrow{d e}$ with endpoints on $p q^{\prime}$ and $p r^{\prime}$ of nonfence $p q^{\prime} c r^{\prime}$ (with $\angle q^{\prime}=\angle r^{\prime}=\pi / 2$ ) are said to have the same orientation if $a$ and $d$ (and $b$ and $e$ ) are on the same edge of $p q^{\prime} c r^{\prime}$, otherwise opposite orientations.

Lemma 6.2. Let $T$ be a trap of case 2 (Section 5) with base $x y$ and length of boundary paths $L$. If the horn of $s \in x y$ entering $T$ self-intersects in opposite directions at a stage less than $L$, then there is a propagation path of length $L$ from $s$ that terminates properly at one dead-end of $T$.

Proof. It suffices to show that the horn of $s$ intersects one boundary path of $T$ and thus contains a dead-end of $T$ at a stage less than $L$. Suppose nonfence $p q^{\prime} c r^{\prime}$ is where the horn first self-intersects in opposite directions. Then two edges, say $\overrightarrow{p_{i} p_{i+1}}$ and $\overrightarrow{p_{j} p_{j+1}}$, of a boundary path $P$ of the horn cross inside $p q^{\prime} c r^{\prime}$. Let $p_{i}$ precede $p_{j}$ in $P$. Suppose $p_{i} \in p q^{\prime}$, then $p_{i+1}, p_{j} \in p r^{\prime}$ and $p_{j+1} \in p q^{\prime}$. Additionally, it is easy to check using incident angles of directed edges that $p_{i} \in p p_{j+1}$ and $p_{j} \in p p_{i+1}$ with $\angle p_{i+1} p_{i} p=\beta$ and $\angle p_{j+1} p_{j} p=\alpha$, as shown in Fig. 6.2(ii), whereas all the other situations (for example, $p_{j+1} \in p p_{i}$ and $p_{i+1} \in p p_{j}$ with $\angle p_{i+1} p_{i} p=\alpha$ and $\left.\angle p_{j+1} p_{j} p=\beta\right)$ are impossible.

Let $P^{\prime}$ be the subpath of $P$ from $\overrightarrow{p_{i} p_{i+1}}$ to $\overrightarrow{p_{j} p_{j+1}}$, and let $\mathcal{R}$ be the region bounded by $P^{\prime}$ (shown shaded in Fig. 6.2(ii)). It is clear that one boundary path of $T$ has some part lying in $\mathcal{R}$ and is coherent to $P^{\prime}$. Let this part be $P^{\prime \prime}$ and let its directed edges be 
labeled in the same way in increasing order as in $P^{\prime}$. Observe that $P^{\prime \prime}$ and $P^{\prime}$ are two forward paths that move closer to each other, as if they are boundary paths of a trap. Moreover, a directed edge of $P^{\prime \prime}$ does not cross another of $P^{\prime}$ of smaller index because of incident angles of directed edges (unless $P^{\prime \prime}$ has already crossed a directed edge of $P^{\prime}$ of the same orientation). Thus, some directed edge of $P^{\prime \prime}$ crosses a directed edge of $P^{\prime}$ of the same orientation since $P^{\prime}$ self-intersects.

Lemma 6.3. The length of each propagation path constructed by Step 3 is less than $12 n$.

Proof. The claim is clearly true when the origin of the propagation path lies on a good segment. So, it suffices to show for each point $s$ lying on a bad segment $x y \subseteq p_{j} q_{j}^{\prime}$ with respect to $p_{j} q_{j}^{\prime} c_{j} r_{j}^{\prime}$ (having $\angle q_{j}^{\prime}=\angle r_{j}^{\prime}=\pi / 2$ ) that there is a properly terminating propagation path with the origin at $s$, the first edge in $p_{j} q_{j}^{\prime} c_{j} r_{j}^{\prime}$, and a length less than $12 n$.

We first review Step 2a on the identification of good segments. Each portion of a good segment identified at each iteration is defined by two endpoints. One is due to the backward path traced from the endpoint of a spoke (such as $q^{\prime}$ in Fig. 5.1). The other is either an endpoint of a spoke or obtained by the extension of an endpoint of the good segment identified in the previous iteration. It is not hard to see that some sequence of vertices mentioned in the previous statement form a backward path with the vertex of the former case (such as $r^{\prime}$ and $r_{2}^{\prime}$ in Fig. 5.1) as the origin. To distinguish each such backward path from those explicitly mentioned in Step $2 a$, we call it an implicit backward path.

First we consider at least one of the endpoints of $x y$, say $x \notin S$, to be a vertex of an implicit backward path, say $P_{r^{\prime}}$, with origin $r^{\prime}$ and in the direction out of $p q^{\prime} c r^{\prime} \subset p q r$. (For example, in Fig. $5.1 x$ can be the endpoint on $p r_{1}^{\prime}$ of the dashed line incident to $r^{\prime}$.) We trace a forward path $P_{s}$ with origin $s$ and parallel to $P_{r^{\prime}}$ until $P_{s}$ and $P_{r^{\prime}}$ have vertices separated by an endpoint of a spoke, or until $P_{s}$ arrives at $q r^{\prime} c p^{\prime}$ (which is adjacent to $p q^{\prime} c r^{\prime}$ ). We discuss the latter with $P_{s}$ terminating at $u \in q r^{\prime}$; we can treat the former similarly. We are clearly done if $q r^{\prime} c p^{\prime}$ is a fence. If not, we are done too because $u \in p^{\prime \prime} r^{\prime} \subset q r^{\prime}$ where $\angle p^{\prime} p^{\prime \prime} r^{\prime}=\beta$ and $P_{s}$ plus $\overrightarrow{u p}^{\prime}$ is a good propagation path; otherwise $u \in q p^{\prime \prime}$ which implies that the backward path with origin $p^{\prime}$ into $q r^{\prime} c p^{\prime}$ traced by Step 2a reaches $x s$, a contradiction to the fact that $x y$ is a bad segment.

Next, we let $x$ and $y$ be vertices of backward paths with origins $x_{0}$ and $y_{0}$ traced in Step 2a. Then the horn of $s$ at a stage no larger than $K$ encounters one of the three situations in Fig. 6.4. Figure 6.4(iii) shows the horn intersecting some good segment, and a propagation path of the required length can clearly be constructed. Fig. 6.4(i) shows the horn entering a trap with $x_{0}$ as an endpoint of its base, and Fig. 6.4(ii) a trap with $x^{\prime}$ as an endpoint of its base. Note that the case of $x^{\prime}$ in Fig. 6.4(ii) is a vertex of some implicit backward path has already been discussed in the previous paragraph.

Let $z$ be a point common to the horn of $s$ and the base of the trap $T$ that the horn enters. Now if $T$ has one dead-end, we are done. This is because a forward path with origin $z$ and parallel to one boundary path of $T$ meets the other boundary path of $T$ and, thus, the horn of $z$ contains the dead-end of $T$. In such a case we can derive one properly terminating propagation path $P_{z}$ with origin $z$ and length bounded by that of the boundary paths of $T$. A propagation path from $s$ to $z$ concatenated to $P_{z}$ gives a properly terminating 


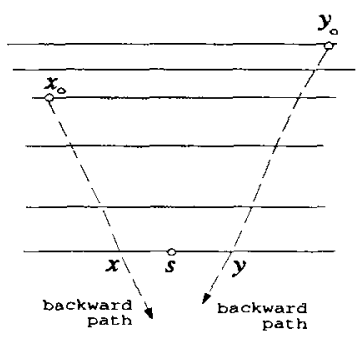

(i)
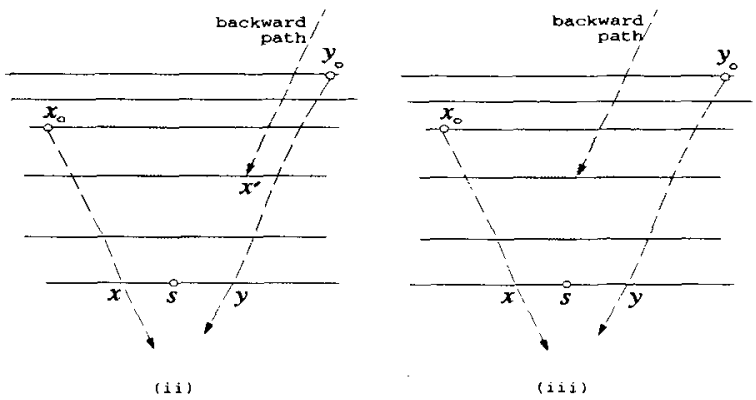

Fig. 6.4. Edges of quadrilaterals are drawn as horizontal line segments here. The horn of $s$ (shaded region) encounters either only bad segments as in (i) and (ii) or some good segments as in (iii).

propagation path of the required length. On the other hand, if $T$ has two dead-ends (case 2 in Section 5), then an extension of results in [17] (Lemma A.3 in the Appendix) plus Lemma 6.2 imply the existence of a propagation path of the required length. Also, if $T$ was not really constructed because one endpoint of its base coincides with a vertex, say $p_{1}$, of $T$, then the horn of $z$ in this case moves only around edges incident to $p_{1}$ and self-intersects at $z$ after one round (by Lemma A.1 in the Appendix), thus a propagation path of the required length that terminates properly at $z$ can be derived.

Lemma 6.4. Step 3 computes less than $34 n$ propagation paths.

Proof. Since a trap has one or two dead-ends, we have in total at most $28 n$ dead-ends by Lemma 5.2 . So Step 3 generates at most $28 n$ propagation paths with origins at deadends. The remaining propagation paths are due to the endpoints of spokes of less than $2 n$ triangles.

\section{Removing Complications}

The next step considers merging of nearby propagation paths. Besides removing redundant portions of paths, i.e., subpaths, this step resolves a complication in triangulating nonfences using edges of propagation paths (in Section 9). We label vertices of nonfence $p q^{\prime} c r^{\prime}$ (with $\angle q^{\prime}=\angle r^{\prime}=\pi / 2$ ) along $p q^{\prime}$ as $p=q_{0}, q_{1}, q_{2}, \ldots, q_{l}=q^{\prime}$ and along $p r^{\prime}$ as $p=r_{0}, r_{1}, r_{2}, \ldots, r_{m}=r^{\prime}$. A neighboring pair of directed edges are two directed edges of the same orientation such that the open segment defined by their tails contains no other tail.

Step 4: Merging Paths. For each nonfence $p q^{\prime} c r^{\prime}$, repeat the following as long as there is a neighboring pair $\overrightarrow{q_{i} r_{i^{\prime}}}$ and $\overrightarrow{q_{j} r_{j^{\prime}}}, i<j$ and $i^{\prime} \neq j^{\prime}$, with $\beta \leq \angle r_{i^{\prime}} q_{j} p \leq \alpha$, or, analogously, a neighboring pair $\overrightarrow{r_{i} q_{i^{\prime}}}$ and $\overrightarrow{r_{j} q_{j^{\prime}}}, i<j$ and $i^{\prime} \neq j^{\prime}$, with $\beta \leq \angle q_{i^{\prime}} r_{j} p \leq \alpha$.

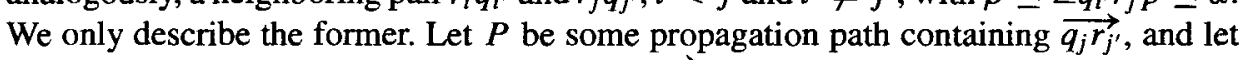
$\boldsymbol{P}^{\prime}$ be the shortest subpath of $P$ starting with $\overrightarrow{q_{j} r_{j^{\prime}}}$ until reaching a vertex $z$, where $z$ can 
be an endpoint of a spoke, or a vertex on an edge of a fence, or a head common to two or more directed edges, or $z=q_{j}$. Remove edges and vertices of $P^{\prime}$, except for its last vertex if shared by other propagation paths. Then, terminate properly the subpath of $P$ before $\overrightarrow{q_{j} r_{j^{\prime}}}$ (if any) by $\overrightarrow{q_{j} r_{i^{\prime}}}$.

Lemma 7.1. All propagation paths still terminate properly with the merging of paths.

Proof. It suffices to consider propagation paths other than $P$. Since each vertex, other than an endpoint of a spoke, does not act as both a head and a tail of different directed edges in a nonfence, vertices removed in one iteration are not involved in other propagation paths. Also, when the last vertex $z$ of $P^{\prime}$ is not removed, it does not result in a bad angle of $\pi$ because it remains incident to either two directed edges or a directed edge and a spoke at both sides of the edge of $\mathcal{T}$ containing $z$. So, all propagation paths still terminate properly.

\section{Untangling Crossings}

For this and the next section, we view each directed edge as an individual with two good angles at its tail, without associating it to a particular propagation path. With reference to nonfence $p q^{\prime} c r^{\prime}$ having Steiner vertices along $p q^{\prime}$ and $p r^{\prime}$ labeled as in Section 7 , the next step is to remove all crossings, in particular, crossings due to directed edges of opposite orientations. It replaces some existing directed edges by new ones while maintaining the following invariants:

I-1. Each edge is assigned an orientation such that both angles at its tails are good.

I-2. Each Steiner vertex is an endpoint of some directed edge.

I-3. No Steiner vertex is a tail of more than one directed edge.

I-4. Directed edges of the same orientation do not cross.

I-5. For $\overrightarrow{q_{i} r_{i^{\prime}}}$ and $\overrightarrow{q_{j} r_{j^{\prime}}}$ with $i<j$ and $i^{\prime}<j^{\prime}$, the region defined by their four endpoints can be subdivided into two good triangles using $r_{i^{\prime}} q_{j}$. Analogous assertion applies to $\overrightarrow{r_{i} q_{i^{\prime}}}$ and $\overrightarrow{r_{j} \vec{q}_{j^{\prime}}}$.

Initially, I-1, I-2, and I-3 are trivially true. Also, I-4 and I-5 are true; otherwise, it is easy to verify that Step 4 did not complete all possible mergings. In Step 5 the next available index from $i$ refers to the smallest index, starting at $i$ and in increasing order, whose corresponding vertex can be used as the head of a new directed edge while maintaining I-4. We say an ordered pair $(a, b)$ is lexicographically smaller than another ordered pair $\left(a^{\prime}, b^{\prime}\right)$ if $a<a^{\prime}$, or $a=a^{\prime}$ and $b<b^{\prime}$.

Step 5: Untangling Crossings. Consider each nonfence $p q^{\prime} c r^{\prime}$ one by one. The following is repeated until all crossings are removed. Each iteration involves $q_{i} r_{i_{1}}$ crossing $r_{j} q_{j_{1}}$, $i<j_{1}$ and $j<i_{1}$, such that $\left(i+j, i_{1}+j_{1}\right)$ is lexicographically the smallest ordered pair.

Case $A: \overrightarrow{q_{i} r_{i_{1}}}$ crosses $\overrightarrow{r_{j} q_{j_{1}}}$ (see Fig. 8.1). There are two symmetrical cases depending on which of $q_{i}$ and $r_{j}$ has good angles. We only discuss the former. For each $\overrightarrow{q_{j}{ }^{\prime} r_{1}}$, $i \leq j^{\prime}<j_{1}$, if $\angle r_{j} q_{j^{\prime}} q^{\prime} \leq \alpha$, we move its head from $r_{i_{1}}$ to $r_{j}$ (i.e., replace $\overline{q_{j^{\prime}} r_{i_{1}}}$ by 

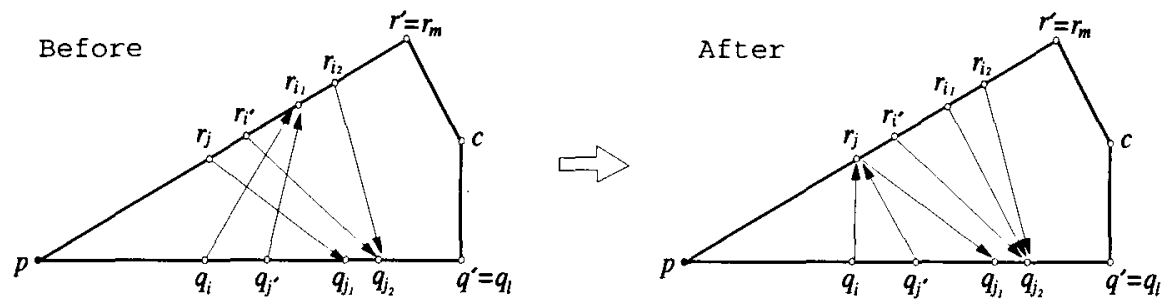

Fig. 8.1. Any directed edge $e$ with an endpoint on $r_{i^{\prime}}$ for $j \leq i^{\prime}<i_{1}$ crosses $\overrightarrow{q_{i} r_{i}}$ by the choice of $\left(i+j, i_{1}+j_{1}\right.$ ). Because of this and I-4, the other endpoint $q_{j_{2}}$ of $e$ is such that $j_{2} \geq j_{1}$, and the orientation of $e$ is opposite to $\overrightarrow{q_{i} r_{i_{1}}}$. A similar statement applies to directed edges such as $\overrightarrow{q_{j^{\prime}} \vec{r}_{i_{1}}}$ for $i \leq j^{\prime}<j$. In the above, $\overrightarrow{r_{i_{1}} q_{j_{2}}}$ is added to maintain I-2 after those edges with the head at $r_{i_{1}}$. have been moved to $r_{j}$.

$\left.\vec{q}_{j^{\prime}} r_{j}\right)$. As a result, if $r_{i_{1}}\left(\neq r_{m}\right)$ is no longer a vertex of any directed edge, we perform the following: if $\angle q_{j_{1}} r_{i_{1}} r_{m} \leq \alpha$, add $\overrightarrow{r_{i_{1}} q_{j_{2}}}$ where $j_{2}$ is the next available index from $j_{1}$, else add $\overrightarrow{q_{j_{1}} r_{i_{1}}}$.

Case B: ${\overrightarrow{i_{1}}}_{q_{i}}$ crosses ${\overrightarrow{q_{j_{1}}}}_{j}$ (see Fig. 8.2). We add $\vec{q}_{i} r_{j}$ if $q_{i}$ is not a tail of any directed edge, else add $\overrightarrow{r_{j} q_{i}}$ if $r_{j}$ is not a tail of any directed edge. Next, if $r_{i_{1}}$ is a head of another directed edge, we are done by removing $\vec{r}_{i_{1}} \vec{q}_{i}$. Symmetrically, if $q_{j_{1}}$ is a head of another directed edge, we are done by removing $\overrightarrow{q_{j_{1}}} \vec{r}_{j}$. Otherwise, move the head $q_{i}$ of all directed edges crossing $\overrightarrow{q_{j_{2}} r_{j}}$, inclusive of $\overrightarrow{r_{i_{1}} \vec{q}_{i}}$, to $q_{j^{\prime}}$ where $j^{\prime}=i+1$.

It is clear that each iteration of either Case A or Case B may introduce new crossings. Still, the process does terminate by removing all crossings. This is because:

(i) The new crossing introduced by say $q_{a} r_{a^{\prime}} \cap r_{b} q_{b^{\prime}}$, for $a<b^{\prime}$ and $b<a^{\prime}$, is such that $a+b>i+j$.

(ii) There are finitely many crossings due to line segments each joining a pair of Steiner vertices.

The next lemma is useful in the verification of the invariants after each iteration of Cases $\mathrm{A}$ and $\mathrm{B}$.

Lemma 8.1. For $\Delta p q_{i} r_{i^{\prime}}$ with $\angle p<\beta$, at least one of the two exterior angles at $q_{i}$ and $r_{i^{\prime}}$ is good.
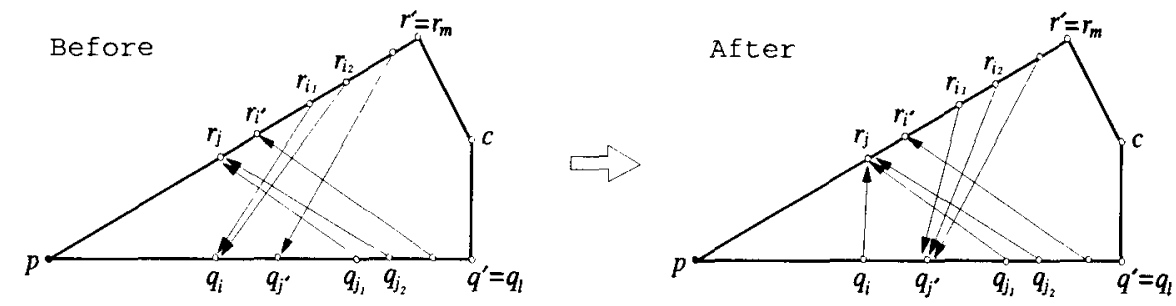

Fig. 8.2. The directed edges shown have orientations enforced by the choice of $\left(i+j, i_{1}+j_{1}\right)$ and I-4. Since neither $r_{i_{1}}$ nor $q_{j_{1}}$ is a head of another directed edge, this iteration removed the crossing between $\overrightarrow{r_{i_{1}} q_{i}}$ and $\overrightarrow{q_{j_{1}}} \vec{r}_{j}$ by moving all edges with the head at $q_{i}$ to $q_{j^{\prime}}$, and adding $\vec{q}_{i} \vec{r}_{j}$. 
Lemma 8.2. Invariants I-1 to I-5 are maintained by each iteration of Case A.

Proof. Refer to Fig. 8.1 for the relative positions of vertices mentioned in the following discussion. We first show that each stated edge when added satisfies I-1. First, consider $q_{i} r_{j}$. Clearly, $\angle q_{i}$ and $\angle r_{j}$ inside $\triangle p q_{i} r_{j}$ are good as they are parts of two good angles at tails of $\overrightarrow{q_{i}} \vec{r}_{i}$ and $\overrightarrow{r_{j} q_{j}}$. As $\angle p<\beta$, Lemma 8.1 implies that one of the two exterior angles of $\Delta p q_{i} r_{j}$ at $q_{i}$ or $r_{j}$ is good, and we are done for $q_{i} r_{j}$. We can now assume, by symmetry, for the remaining proof that $\vec{q}_{i} \vec{r}_{j}$ is added. We can check similarly that each addition of directed edges $\overrightarrow{q_{j^{\prime}} r_{j}}$ (obtained from $\overrightarrow{q_{j^{\prime}} \vec{r}_{i_{1}}}$ ), $\overrightarrow{r_{i_{1}} q_{j_{2}}}$, or $\overrightarrow{q_{j_{1}} r_{i_{1}}}$ satisfies I-1. This completes the verification for I- 1 .

The moving of heads of $\overrightarrow{q_{j^{\prime}} r_{i_{1}}}$ maintains I-4 because $\angle r_{j} q_{j^{\prime}} q^{\prime} \leq \alpha$ for some $j^{\prime}$ implies that it is also true for smaller $j^{\prime}$. (So this moving can be carried out in increasing order of the indices of tails.) By construction, $\overrightarrow{r_{i_{1}} \vec{q}_{j_{2}}}$ when added still maintains I-4. Next, notice that if $\angle q_{j_{1}} r_{i_{1}} r_{m}$ is bad, $\overrightarrow{q_{j^{\prime}} r_{i_{2}}}$ with $i<j^{\prime} \leq j_{1}$ and $i_{1}<i_{2}$ cannot exist because $\overrightarrow{q_{i} r_{i_{1}}}$ satisfies I-5 before the iteration. So, adding $\overrightarrow{q_{j_{1}}}{\overrightarrow{i_{1}}}_{i_{1}}$ does not violate I-4 (and I-3), and the invariant is maintained. Invariants I-2 and I-3 are clearly valid.

In the following verification of I-5, we mention only one of the six angles involved since the remaining five can easily be checked to be good. Consider $\overrightarrow{q_{i} \vec{r}_{j}}$ with other $\overrightarrow{q_{f} r_{f^{\prime}}}$ where $j \neq f^{\prime}$ (and $i \neq f$ ). If $i>f$, then $\angle q_{i} r_{f^{\prime}} r_{j}=\angle q_{i} r_{f^{\prime}} r_{i_{1}}$, which is good because $\overrightarrow{q_{f} r_{f}^{\prime}}$ with $\overrightarrow{q_{i} r_{i}}$ satisfies I-5. Next, if $i<f<j_{1}$ and $f^{\prime}=i_{1}$, then $\angle r_{j} q_{f} q^{\prime}>\alpha$ implies that $\angle q_{f} r_{j} r_{i_{1}}<\alpha$ (Lemma 8.1). If $i<f<j_{1}$ and $f^{\prime}>i_{1}, \angle q_{f} r_{j} r_{f^{\prime}}<\angle q_{f} r_{i_{1}} r_{f^{\prime}} \leq \alpha$ by the fact that $\overrightarrow{q_{i} r_{i_{1}}}$ with $\overrightarrow{q_{f} r_{f^{\prime}}}$ satisfies I-5. Lastly, if $i<j_{1} \leq f$, then $\angle q_{f} r_{j} r_{f^{\prime}} \leq \alpha$ because of $\overrightarrow{r_{j} q_{j_{1}}}$. Notice that the argument simply applies I-5 on existing edges sharing endpoints with $\vec{q}_{i} \vec{r}_{j}$ to show that $\vec{q}_{i} \vec{r}_{j}$ satisfies I-5. The same approach applies to other newly added edges $\overrightarrow{q_{j^{\prime}} r_{j}}, \overrightarrow{r_{i_{1}} q_{j_{2}}}$ and $\overrightarrow{q_{j_{1}} r_{i_{1}}}$. This completes the proof.

Lemma 8.3. Invariants I-1 to I-5 are maintained by each iteration of Case $B$.

Proof. Refer to Fig. 8.2 for the relative positions of vertices mentioned in the following discussion. We begin with I-1. First we show that all the eight angles at the endpoints of $q_{i} r_{j}$ and $r_{i_{1}} q_{j_{1}}$ are good, so directed edges based on them always satisfy I-1. The two exterior angles of $\Delta p q_{j_{1}} r_{i_{1}}$ at $r_{i_{1}}$ and $q_{j_{1}}$ are good because they are only parts of some good angles at tails of $\vec{r}_{i_{1}} \vec{q}_{i}$ and $\vec{q}_{j_{1}} r_{j}$. Next looking at $\Delta q_{i} r_{i_{1}} r_{j}$, we have good $\angle r_{j}$ followed from the good exterior angle at $r_{i_{1}}$ because of ${\overrightarrow{i_{i}}}_{q_{i}}$. Similarly, we have good $\angle r_{j} q_{i} q_{j j_{1}}$. Now with these four good angles of interest as exterior angles of $\Delta p q_{i} r_{j}$ and $\Delta p q_{j_{1}} r_{i_{1}}$, we can easily deduce that the other four (interior) angles of interest are also good.

Next, we consider each $\overrightarrow{r_{i}} \vec{q}_{i}$ for $i_{2} \geq i_{1}$ that is moved to $\overrightarrow{r_{i_{2}} q_{j^{\prime}}}$ where $j^{\prime}=i+1$. For $\overrightarrow{r_{i} q_{i}}$, we can indeed have $\overrightarrow{r_{i} q_{j^{\prime}}}$ as it is within the wedge defined by $\overrightarrow{r_{i} q_{i}}$ and $\overrightarrow{r_{i_{1}} q_{j_{1}}}$. Similarly, each $\overrightarrow{i_{i_{2}} q_{j}}$ for $i_{2} \neq i_{1}$ satisfies I-1. This completes verifying $\mathrm{I}-1$.

Invariants I-2, I-3, and I-4 are valid by construction. We next verify I-5, as in the previous lemma, by checking one of the six angles involved. Since all four angles at the endpoints of $q_{i} r_{j}$ are good, $\vec{q}_{i} \vec{r}_{j}$ or $\vec{r}_{j} \vec{q}_{i}$ can never be involved to invalidate I-5. Next, consider $\overrightarrow{r_{i_{2}} q_{j^{\prime}}}$, for $i_{2} \geq i_{1}$, with other $\overrightarrow{r_{g} q_{g^{\prime}}}$ where $j^{\prime} \neq g^{\prime}$. If $g^{\prime}=i$, we have $\angle r_{i_{2}} q_{i} q_{j^{\prime}}<\angle r_{j} q_{i} q_{j^{\prime}} \leq \alpha$. Now suppose $g^{\prime} \neq i$. Since $\overrightarrow{r_{i_{2}} q_{j^{\prime}}}$ has the same tail as $\overrightarrow{r_{i_{2}} q_{i}}$, I-5 
is maintained when $i_{2}>g$ because of the fact that $\overrightarrow{r_{i_{2}} q_{i}}$ with $\overrightarrow{r_{g} q_{g^{\prime}}}$ satisfies I-5. Lastly, for $i_{2}<g$, we have $\angle r_{g} q_{j^{\prime}} q_{g^{\prime}}<\angle r_{j} q_{j^{\prime}} q_{g^{\prime}} \leq\left(\pi-\angle r_{j} q_{j_{1}} p\right) \leq \alpha$. This completes the proof.

\section{Triangulating Quadrilaterals}

For each nonfence $p q^{\prime} c r^{\prime}$, any directed edges in its interior do not cross. Thus, they subdivide $p q^{\prime} c r^{\prime}$ into regions of three, four, or five sides. All regions except possibly the one containing $c$ have good triangulations, i.e., triangulations with no bad angles. We must nevertheless handle the region containing $c$ first by merging it with nearby regions. Hence, some directed edges are just guides in the construction and may not be in the final triangulation.

Step 6: Triangulating Quadrilaterals. Fences and quadrilaterals without subdivision points can easily be triangulated with good triangles; refer to Lemma 4.1. We next consider each nonfence $p q^{\prime} c r^{\prime}$ with $\angle q^{\prime}=\angle r^{\prime}=\pi / 2$. Label its faces from $c$ to $p$ by $R_{1}, R_{2}, \ldots, R_{m^{\prime}}$. Starting with $\mathcal{R}=\emptyset$ and $k=0$, we then repeat incrementing $k$ and including $R_{k}$ into $\mathcal{R}$ until we can produce a good triangulation for $\mathcal{R}=\cup_{i=1}^{k} R_{i}$. Then for the remaining regions $R_{j}$, for $j=k+1, k+2 \ldots, m^{\prime}$, of three or four sides, we triangulate each in a straightforward way.

The following two lemmas show that Step 6 can indeed produce a good triangulation for $p q^{\prime} c r^{\prime}$. Let the vertices along $p q^{\prime}$ be sorted as $p=q_{0}, q_{1}, \ldots, q_{l}=q^{\prime}$ and along $p r^{\prime}$ as $p=r_{0}, r_{1}, \ldots, r_{m}=r^{\prime}$.

Lemma 9.1. Each region $R_{k}$ for $k=2,3, \ldots, m^{\prime}$ has a good triangulation.

Proof. If $R_{k}$ is of three sides, then it is $p q_{1} r_{1}, q_{i} q_{i+1} r_{j}$, or $r_{j} r_{j+1} q_{i}$. With the directed edge based on $q_{1} r_{1}, \Delta p q_{1} r_{1}$ is clearly good, and, $\Delta q_{i} q_{i+1} r_{j}$ is good because $\vec{q}_{i} \vec{r}_{j}$ or $\overrightarrow{q_{i+1} r_{j}}$ exists by I-3 and $\angle q_{i}$ or $\angle q_{i+1}$ is thus between $\beta$ and $\alpha$. Similarly, $\Delta r_{j} r_{j+1} q_{i}$ is good. Now, if $R_{k}$ is of four sides, say $q_{i} q_{i+1} r_{j+1} r_{j}$, then it is supported by two directed edges based on $q_{i} r_{j}$ and $q_{i+1} r_{j+1}$. If they are of opposite orientations, then $q_{i} q_{i+1} r_{j+1} r_{j}$ has two opposite angles each measuring between $\beta$ and $\alpha$. So we have a good triangulation for the region by adding the diagonal not incident to these good angles. If they are of the same orientation, a good triangulation is ensured by $\mathrm{I}-5$. We are done as $R_{k}$ has either three or four sides by $\mathrm{I}-2$.

Lemma 9.2. $A k \leq m^{\prime}$ exists such that $\mathcal{R}=\bigcup_{i=1}^{k} R_{i}$ has a good triangulation.

Proof. We consider successively larger $\mathcal{R}$ beginning with $\mathcal{R}=R_{1}$, then $\mathcal{R}=R_{1} \cup R_{2}$ and so on. For each one, we test $\mathcal{R}$ for a good triangulation by joining $c$ with Steiner vertices on the boundary of $\mathcal{R}$ so as to subdivide $\angle q_{l} c r_{m}$ further. As we will see, a good $\mathcal{R}$ is found once $\angle q_{l} c r_{m}$ can be subdivided into good angles. The details follow:

Initially, $\mathcal{R}=R_{1}$ which is (1) $q_{l} c r_{m}$, (2) $q_{l-1} q_{l} c r_{m}$, (3) $q_{l} c r_{m} r_{m-1}$, or (4) $q_{l-1} q_{l} c r_{m} r_{m-1}$. 
For (1), we just go on to enlarge $\mathcal{R}$ since $\angle c$ is bad. For (2) and (3), we add $c q_{l-1}$ and $c r_{m-1}$, respectively, to subdivide $\angle c$. We are done if we have only good angles around $c$. For (4), if $\left|c q_{l-1}\right| \leq\left|c r_{m-1}\right|$ we add $c q_{l-1}$, else add $c r_{m-1}$, resulting in a region yet to be triangulated. If $\angle c$ of this region is good, we are done by completing the triangulation with the diagonal not incident to $c$; otherwise, we use the other diagonal to subdivide $\angle c$ further and are done if all angles around $c$ are good.

To enlarge $\mathcal{R}$, we remove the directed edge supporting the current $\mathcal{R}$. In general, we have just removed an edge say $q_{i+1} r_{j+1}$ to obtain a part of $\mathcal{R}$ which has yet to be triangulated. This is $q_{i} q_{i+1} c r_{j+1}, q_{i+1} c r_{j+1} r_{j}$, or $q_{i} q_{i+1} c r_{j+1} r_{j}$, which is handled analogously as in (2)-(4).

It is easy to see that the process terminates, producing only good angles around $c$. We next verify that only good triangles are produced. Suppose the process terminates with (2) creating $\Delta q_{i} c r_{j+1}$ (and $\Delta q_{i} q_{i+1} c$ ) from $q_{i} q_{i+1} c r_{j+1}$. Consider $q_{i} q_{l} c r_{j+1}$. Since $\angle q_{l} c r_{j+1}>\alpha$ and $\angle q_{l}=\pi / 2$, both $\angle q_{i}$ and $\angle r_{j+1}$ are good. So $\triangle q_{i} c r_{j+1}$ is good. Symmetrically, the triangle created last due to (3) is also good. For (4), the process terminates either similarly to (2) and (3) or with a region, say (without loss of generality) $q_{i} c r_{j+1} r_{j}$, subdivided into $\Delta q_{i} c r_{j+1}$ and $\Delta q_{i} r_{j+1} r_{j}$. Note that $\Delta q_{i} c r_{j+1}$ is good as $q_{i} q_{l} c r_{j+1}$ has $\angle c>\alpha$ and $\angle q_{l}=\pi / 2$, and $\Delta q_{i} r_{j+1} r_{j}$ is good as $\angle r_{j}$ is good by our choice of $c q_{i}$ to triangulate $q_{i} q_{i+1} c r_{j+1} r_{j}$, and $\angle q_{i}$ and $\angle r_{j+1}$ are good by the existence of directed edges based on $q_{i} r_{j}$ and $q_{i+1} r_{j+1}$, respectively.

The rest of the triangles resulting from the above are of the form $c q_{i^{\prime}} q_{i^{\prime}+1}$ or the symmetrical form $c r_{j^{\prime}} r_{j^{\prime}+1}$. For $\Delta c q_{i^{\prime}} q_{i^{\prime}+1}, \angle q_{i^{\prime}}$ and $\angle c$ are clearly good as they are acute, and $\angle q_{i^{\prime}+1}$ is good from the following: $\angle q_{i^{\prime}+1} c r_{m}>\alpha$ because a directed edge incident to $q_{i^{\prime}+1}$ was removed to enlarge $\mathcal{R} ; \angle q_{i^{\prime}+1} c q_{l}<\pi-\angle q_{i^{\prime}+1} c r_{m}<\pi-\alpha$ and $\angle c q_{i^{\prime}+1} q_{i^{\prime}}=\angle q_{i^{\prime}+1} c q_{l}+\angle q_{i^{\prime}+1} q_{l} c<\frac{3}{2} \pi-\alpha=\alpha$.

We have now established the following theorem:

Theorem 9.3. Triangulating a plane geometric graph $\mathcal{G}=(S, E)$ of $|S|=n$ vertices and $|E|=O(n)$ edges using angles no larger than $\frac{3}{4} \pi$ requires $O\left(n^{2}\right)$ Steiner vertices.

\section{Implementing Construction}

In this section we describe an efficient algorithm to implement the above constructive proof. Also, we discuss ways to avoid some redundant Steiner vertices, and extend the construction to a better angle bound of $\alpha=\frac{11}{15} \pi$. We assume that each point coordinate can be stored in a constant amount of storage and that basic geometric operations, such as projecting a point onto a line, can be carried out in a constant amount of time.

Theorem 10.1. Triangulating a plane geometric graph $\mathcal{G}=(S, E)$ of $|S|=n$ vertices and $|E|=O(n)$ edges using angles no larger than $\frac{3}{4} \pi$ requires $O\left(n^{2}\right)$ storage and $O\left(n^{2} \log n\right)$ time.

Proof. From $\mathcal{G}$, we can first compute in $O\left(n^{2} \log n\right)$ time the triangulation $\mathcal{T}$ that minimizes its maximum angle over all triangulations of $\mathcal{G}$ [11] and store it in a quad- 
edge data structure of $O(n)$ storage [13]. If the maximum angle of $\mathcal{T}$ is no larger than $\frac{3}{4} \pi$, then we are done. Otherwise, we proceed to refine $\mathcal{T}$ with the construction given in Sections 4-9. To perform those steps efficiently, we maintain for each edge of $\mathcal{T}$ a sorted list of $O(n)$ vertices of propagation paths on the edge, and we link up vertices with pointers that act as directed edges of propagation paths. Also, we keep some general information about each quadrilateral edge (such as its type as a fence or nonfence, its good and bad segments, etc.) and about each vertex (such as its type as a dead-end or an endpoint of a spoke, its outgoing pointer, etc.). All in all, these structures requires $O\left(n^{2}\right)$ storage.

With the above, Steps 1-3 are straightforward. The splitting of an edge of $\mathcal{T}$ into edges of quadrilaterals, and the subsequent walking from one quadrilateral to an adjacent one can each be done in constant time with the quad-edge data structure. The checking of vertices within a maw, and the insertion of a vertex to an edge of $\mathcal{T}$ (or a quadrilateral), can be done in logarithmic time. Therefore, Steps $1-3$ are bounded by $O\left(n^{2} \log n\right)$ time. Step 4 is simple by first separating directed edges of opposite orientations into two sets and then working on each set in say increasing order of neighboring pairs. This step thus takes $O\left(n^{2}\right)$ time.

Next, Step 5 is slightly involved. We discuss it with notation introduced in Section 8 and with reference to nonfence $p q^{\prime} c r^{\prime}$ of $O(n)$ Steiner vertices. Note first that the sum $i+j+i_{1}+j_{1}$ (from the lexicographically smallest ordered pair) is increasing in successive iterations because of I-4. Thus, the bound of $O(n)$ on the sum also bounds the number of iterations. Note second that the next available index along $p q^{\prime}$ (or $p r^{\prime}$ ) in Case A is also increasing in successive iterations. The reason is that each vertex $v$ that we passed in a search for the next available index remains unavailable because of blocking by other edges, or $v$ becomes unblocked by the removal of directed edges but is now out of range of the searching. Thus, a careful implementation of Step 5 needs only $O(n)$ time in locating vertices along $p q^{\prime}$ (or $p r^{\prime}$ ) by marching across $p q^{\prime}$ and $p r^{\prime}$ once in some coherent way. With these notes, we can implement Step 5 to run in $O(n)$ time by maintaining for each Steiner vertex a list of directed edges with heads at the vertex. Each list stores elements in increasing order of indices of tails, and is a queue that allows a removal of an element or an appending of another queue in constant time. So, crossings of directed edges inside all quadrilaterals can be removed in $O\left(n^{2}\right)$ time.

Lastly, Step 6 is straightforward and runs in time linear to the number of vertices in the resulting triangulation.

Reducing Steiner Vertices. The above construction generates considerably many Steiner vertices, though it achieves the worst-case optimal bound within a constant factor. We see, in the following, a modified construction that avoids many redundant Steiner vertices. We start with Step 1. Instead of subdividing each $\Delta p q r$, with the largest angle at $q$, into three quadrilaterals, we subdivide it into two or four triangles as follows. Also note that a fence now has three edges (with only one is a spoke).

Case $a: \angle q>\alpha$. Subdivide $\triangle p q r$ into $\triangle p q q^{\prime}$ and $\Delta r q q^{\prime}$ where $q^{\prime} \in p r$ and $\angle p q q^{\prime}=$ $\angle r q q^{\prime}$. We treat both triangles as nonfences and $q q^{\prime}$ as a spoke.

Case $b: 2 \beta \leq \angle q \leq \alpha$. Same as Case a. However, mark $\triangle p q q^{\prime}$ as a fence if $\angle p \geq \beta$ else as a nonfence, and similarly mark $\Delta r q q^{\prime}$ according to $\angle r$. 
Case $c: \angle q<2 \beta$. Subdivide $\Delta p q r$ into $\Delta p r^{\prime} q^{\prime}, \Delta q p^{\prime} r^{\prime}, \Delta r q^{\prime} p^{\prime}$, and $\Delta p^{\prime} q^{\prime} r^{\prime}$ where $p^{\prime}, q^{\prime}, r^{\prime}$ are the three perpendicular projections of the center of the inscribing circle of $\Delta p q r$ onto $q r, r p, p q$, respectively. Also, treat edges $p^{\prime} q^{\prime}, q^{\prime} r^{\prime}$, and $r^{\prime} p^{\prime}$ as spokes, and mark each of the triangles (excluding $\Delta p^{\prime} q^{\prime} r^{\prime}$ ) as a fence if its angle opposite its spoke is no less than $\beta$, else as a nonfence.

With this subdivision we then plan and set up traps in Steps $2 \mathrm{a}$ and $2 \mathrm{~b}$. Then Step 3 generates propagation paths selectively for dead-ends and endpoints of spokes-so endpoints of spokes are just like dead-ends that may or may not be Steiner vertices in the final construction. A dead-end as before originates a propagation path if it terminates some propagation paths, whereas an endpoint of a spoke originates a propagation path in the following situations. A $q^{\prime}$ of Case a always originates a propagation path. A $q^{\prime}$ of Case $\mathrm{b}$ and $p^{\prime}, q^{\prime}, r^{\prime}$ of Case $\mathrm{c}$ each originates a propagation path if it terminates some other propagation paths, or if the two smaller triangles in $\Delta p q r$ sharing this endpoint have Steiner vertices on their boundaries. (Other refined rules in selecting vertices for propagation are omitted in this paper for simplicity in the presentation.)

Step 4 as before removes unnecessary subpaths. Note that each spoke in a triangle can be considered as a directed edge in either orientation since all four angles defined by its two endpoints with edges of the triangle are good. Spokes are not involved in merging. Next, Step 5 remains the same. Finally, Step 6 works in a similar way as before: for a nonfence, we just use the straightforward method in Lemma 9.1 (treating each spoke as a directed edge of either orientation); otherwise, we have a fence that can be solved with Lemma 4.1. It is easy to check that $\Delta p^{\prime} q^{\prime} r^{\prime}$ of Case $\mathrm{c}$ is good; each of its angles is just a half of the sum of two angles of $\Delta p q r$. However, there is one minor problem- $q^{\prime}$ in Case b or $p^{\prime}, q^{\prime}, r^{\prime}$ in Case c which are used in Step 6 may not be Steiner vertices in the construction as mentioned. Nevertheless, a simple shifting of edges can remedy the problem in all cases; see Fig. 10.1.

Reducing the Angle Bound. The angle bound of $\alpha=\frac{3}{4} \pi$ can be improved slightly to $\alpha=\frac{11}{15} \pi$ (i.e., $132^{\circ}$ ), but with a larger constant in the quadratic bound on vertex size. In this case, $\beta=\frac{4}{15} \pi$ (i.e., $48^{\circ}$ ). All results developed starting from Section 4 are valid except for Lemmas $4.1,8.1$ (with $\angle p<2 \beta$ ), and 9.2. We need not worry about Lemma 9.2 since it is no longer relevant to the above modified construction. As for the other two lemmas, a problem arises when $\pi-2 \beta<\angle p<2 \beta$. One way to resolve this is to

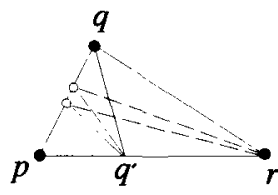

(i)

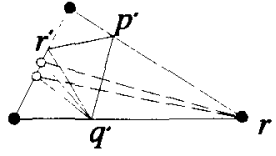

(ii)

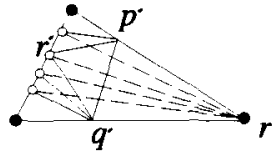

(iii)

Fig. 10.1. Vertex $q^{\prime}$ of Case b (i), $p^{\prime}, q^{\prime}, r^{\prime}$ of Case c (ii), and $p^{\prime}, q^{\prime}$ of Case c (iii) are not Steiner vertices in the construction. Edges incident to these vertices are shifted to $r$ as shown by dashed line segments. All new angles that resulted from the shifting can be checked to be good using the simple extension of Lemma 8.1 to $\angle p<2 \beta$. 
perform the following, before Step 1: for each $\Delta p q r$ of $\mathcal{T}$ with the largest angle between $\pi-2 \beta$ and $2 \beta$, we subdivide it by a new vertex $s$ inside $p q r$ into triangles with angles at $s$ equal to $\frac{2}{3} \pi$. The existence of such a subdivision can be verified as follows. Let $\angle q \geq \angle p \geq \angle r$. We can identify an interior point $s^{\prime}$ on $q r$ such that $\angle p s^{\prime} r=\frac{2}{3} \pi$ since $\angle p+\angle q>\frac{2}{3} \pi$. Hence, we can draw a circle through $p, s^{\prime}, r$ whose arc inside $\triangle p q r$ represents the loci of points forming angles of $\frac{2}{3} \pi$ with $p r$. Note that $\angle q t r$ is $\pi$ when $t=s^{\prime}$, and decreases as $t$ moves along the arc toward the intersection point $s^{\prime \prime}$ of the arc with $p q$. Since $\angle q t r<\frac{2}{3} \pi$ when $t=s^{\prime \prime}$ as $\angle q>\pi / 3$, some point $s$ along the arc is such that $\angle q s r=\frac{2}{3} \pi$.

Corollary 10.2. Triangulating a plane geometric graph $\mathcal{G}=(S, E)$ of $|S|=n$ vertices and $|E|=O(n)$ edges using angles no larger than $\frac{11}{15} \pi$ requires $O\left(n^{2}\right)$ storage and $O\left(n^{2} \log n\right)$ time.

\section{Concluding Remarks}

This paper shows that for any plane geometric graph a conforming triangulation with a quadratic bound on its vertex set and $\frac{3}{4} \pi$ bound on its angles exists. It is possible to extend the result to a slightly better bound on angles, but with a larger constant factor in the quadratic bound on the vertex set. This paper mentions such an improvement for an angle bound of $\frac{11}{15} \pi$. On the whole, it improves the result of Mitchell [17] by reducing a logarithmic factor on the bound on the vertex set and by reducing at least $\pi / 8$ on the bound on angles. The new bound on the vertex set is asymptotically optimal and can be computed in slightly more than quadratic time. The computation is simple and practical, without introducing many unnecessary vertices. On the other hand, it remains open whether the bound on angles can be reduced further.

The main idea of the paper is the control of the lengths of propagation paths using fences and traps. There is a similar idea in [10] where the corresponding notion is termed walls. The current paper is, nevertheless, much more complex and has a number of new strategies to address issues on the number of fences, traps, and crossings of propagation paths. It remains interesting to see whether some of these ideas and strategies can be applied to other triangulation problems.

\section{Acknowledgments}

I would like to thank Scott Mitchell for providing details of his paper [17], and I am grateful to one anonymous referee for many suggestions on improving the presentation of the paper.

\section{Appendix. Analyzing Step 3}

We prove again some important results of Mitchell [17]; our new proofs are necessary because those results are applied here to a more general setting. For a horn of $s$, its center path is the sequence of line segments where each connects the midpoints of the maws of 


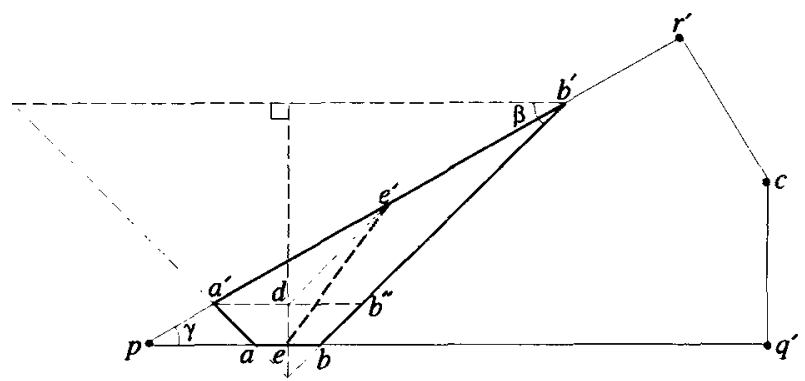

Fig. A.1. The part of the horn of $s$ inside $p q^{\prime} c r^{\prime}$ is $a b b^{\prime} a^{\prime}$. That is, the horn of $s$ arrives at $a b \subset p q^{\prime}$ and then extends to $a^{\prime} b^{\prime} \subset p r^{\prime}$. The midpoint of $a b$ and $a^{\prime} b^{\prime}$ are $e$ and $e^{\prime}$, respectively. Point $b^{\prime \prime} \in b b^{\prime}$ is such that $a^{\prime} b^{\prime \prime}$ is parallel to $a b$, and $d \in a^{\prime} b^{\prime \prime}$ is such that $d e$ is perpendicular to $a b$. Notice that $e^{\prime} d$ is parallel to $b^{\prime} b^{\prime \prime}$.

two consecutive stages, starting at $s$. Each midpoint mentioned is also termed a center path point. The length of a line segment is the distance of its two endpoints, and the length of a center path is the sum of the lengths of its line segments. The maw width of a maw is the distance between its two endpoints. For our following discussion, horns refer to those that do not intersect endpoints of spokes; otherwise our analysis of Step 3 is trivial.

Lemma A.1. For a point s on an edge of $\mathcal{T}$, the maw width of its horn at some stage is greater than the length of the center path up to that stage.

Proof. It suffices to show that the increase in the maw width from one stage to the next is no less than the increase in the length of the center path. Referring to Fig. A.1, we show $\left|a^{\prime} b^{\prime}\right|-|a b| \geq\left|e e^{\prime}\right|$. Note that the case of stage 0 is such that $a=b=e$. We have $|a b|+|d e|<\left|a^{\prime} b^{\prime \prime}\right|$ from simple observation, and $|d e|+\left|d e^{\prime}\right|>\left|e e^{\prime}\right|$ from the triangle inequality. So, we just need to show that $\left|a^{\prime} b^{\prime}\right|-\left|a^{\prime} b^{\prime \prime}\right| \geq\left|d e^{\prime}\right|$. The law of sines for $\Delta a^{\prime} d e^{\prime}$ gives $\left|d e^{\prime}\right|=\left|a^{\prime} e^{\prime}\right| \sin \gamma / \sin \alpha=\frac{1}{2}\left|a^{\prime} b^{\prime}\right| \sin \gamma / \sin \alpha$, and for $\Delta a^{\prime} b^{\prime \prime} b^{\prime}$ it gives $\left|a^{\prime} b^{\prime \prime}\right|=\left|a^{\prime} b^{\prime}\right| \sin (\beta-\gamma) / \sin \alpha$. So,

$$
\begin{aligned}
\frac{\left|a^{\prime} b^{\prime}\right|-\left|a^{\prime} b^{\prime \prime}\right|}{\left|d e^{\prime}\right|} & =\frac{2 \sin \alpha}{\sin \gamma}\left(1-\frac{\sin (\beta-\gamma)}{\sin \alpha}\right) \\
& =\frac{2}{\sin \gamma}(\sin \beta-\sin \beta \cos \gamma+\cos \beta \sin \gamma) \\
& >\frac{2}{\sin \gamma}(\cos \beta \sin \gamma) \\
& =2 \cos \beta>1 .
\end{aligned}
$$

Let $T$ be a trap having two distinct dead-ends (case 2 in Section 5) with base $x y$. For a point $z \in x y$, it is clear that the horn of $z$ into $T$ moves closer in successive stages to both boundary paths of $T$. This notion of closeness can be formalized from the concept of the inverse horn [17]. For our purposes, we define an inverse horn as the region between two coherent forward paths (in a given sequence of nonfences) where the good angles of $\beta$ at the tails of their directed edges are inside the region. The notions of maw, maw 


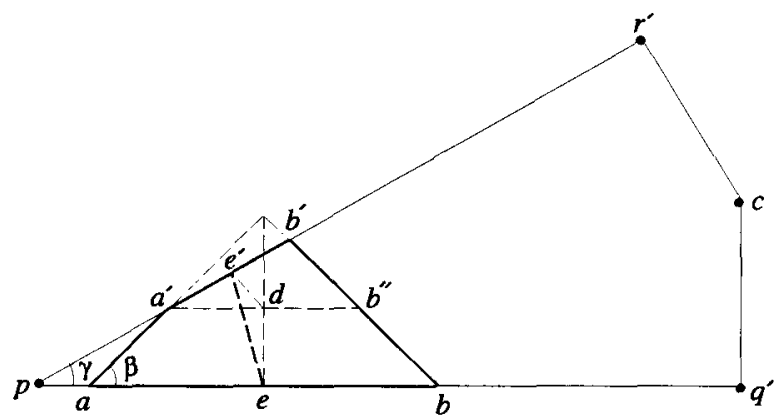

Fig. A.2. Region $a b b^{\prime} a^{\prime}$ is the part of the inverse horn inside $p q^{\prime} c r^{\prime}$. The inverse horn arrives at $a b \subset p q^{\prime}$ and then extends to $a^{\prime} b^{\prime} \subset p r^{\prime}$. The midpoint of $a b$ and $a^{\prime} b^{\prime}$ are $e$ and $e^{\prime}$, respectively. Point $b^{\prime \prime} \in b b^{\prime}$ is such that $a^{\prime} b^{\prime \prime}$ is parallel to $a b$, and $d \in a^{\prime} b^{\prime \prime}$ is such that $d e$ is perpendicular to $a b$. Notice that $e^{\prime} d$ is parallel to $b^{\prime} b^{\prime \prime}$.

width, center path, and boundary paths for a horn are extended analogously to an inverse horn. As mentioned, the maw width of an inverse horn decreases (rather than increases) from one stage to the next. When the maw width is negative, the two boundary paths of the inverse horn have crossed.

Lemma A.2. We consider the inverse horn defined by coherent forward paths originated at $w$ and $z$. Initially, $l=|w z|$ is the maw width. Then the maw width $l^{\prime}$ at some later stage is such that $l-l^{\prime}>\frac{4}{5} l_{\mathrm{c}}$ where $l_{\mathrm{c}}$ is the length of the center path up to that stage.

Proof. Analogous to the previous lemma, it suffices to show that, from one stage to the next, the decrease in maw width is at least four-fifths the increase in the center path length. Referring to Fig. A.2, we show in the following that $|a b|-\left|a^{\prime} b^{\prime}\right|>\frac{4}{5}\left|e e^{\prime}\right|$. The law of sines for $\triangle a^{\prime} d e^{\prime}$ gives $\left|d e^{\prime}\right|=\frac{1}{2}\left|a^{\prime} b^{\prime}\right| \sin \gamma / \sin \beta$, and for $\triangle a^{\prime} b^{\prime \prime} b^{\prime}$ it gives $\left|a^{\prime} b^{\prime \prime}\right|=\left|a^{\prime} b^{\prime}\right| \sin (\pi-\beta-\gamma) / \sin \beta=\left|a^{\prime} b^{\prime}\right| \sin (\beta+\gamma) / \sin \beta$. So,

$$
\frac{\left|a^{\prime} b^{\prime \prime}\right|-\left|a^{\prime} b^{\prime}\right|}{\left|d e^{\prime}\right|}=\frac{2}{\sin \gamma}(\sin (\beta+\gamma)-\sin \beta)
$$

and, for $0<\gamma<\beta=\pi / 4,\left(\left|a^{\prime} b^{\prime \prime}\right|-\left|a^{\prime} b^{\prime}\right|\right) /\left|d e^{\prime}\right|$ is decreasing in $\gamma$. This means

$$
\frac{\left|a^{\prime} b^{\prime \prime}\right|-\left|a^{\prime} b^{\prime}\right|}{\left|d e^{\prime}\right|}>\frac{4}{5}
$$

so the claim is proved because $|a b|>\left|a^{\prime} b^{\prime \prime}\right|+|d e|$ and $|d e|+\left|d e^{\prime}\right|>\left|e e^{\prime}\right|$.

With the above lemmas, we next complete the argument of Lemma 6.3 using the next result, which implies the existence of a properly terminating propagation path with origin $z$ and length $O(n)$. 
Lemma A.3. Let $z$ be a point on the base of a trap $T$ having two distinct dead-ends (case 2 in Section 5). Then the horn of $z$ inside $T$ either self-intersects or intersects at least one boundary path of $T$ at a stage no larger than the length of the boundary paths of $T$.

Proof. Let $p q$ be the edge intersected three times by each boundary path of $T$ (so $p q$ contains the dead-ends of $T$ ). For the proof, it suffices to consider the horn $\mathcal{H}_{z}$ of $z_{1}$ inside $T$ where $z_{1}$ is some point on the first intersection of the horn of $z$ and $p q$. Assuming $\mathcal{H}_{z}$ does not self-intersect inside $T$, we prove that $\mathcal{H}_{z}$ intersects a boundary path of $T$. Each boundary path of $T$ with one boundary path of $\mathcal{H}_{z}$ forms an inverse horn. Refer to Fig. A.3; the inverse horn $\mathcal{I}$ of interest has initial maw $z_{1} w_{1}$ where $w_{1}$ (on a boundary path of $T$ ) lies on $z_{1} v$ and $v$ is some point on the second intersection of $\mathcal{H}_{z}$ with the line through pq. (In Fig. A.3, $v$ is $z_{2}$.)

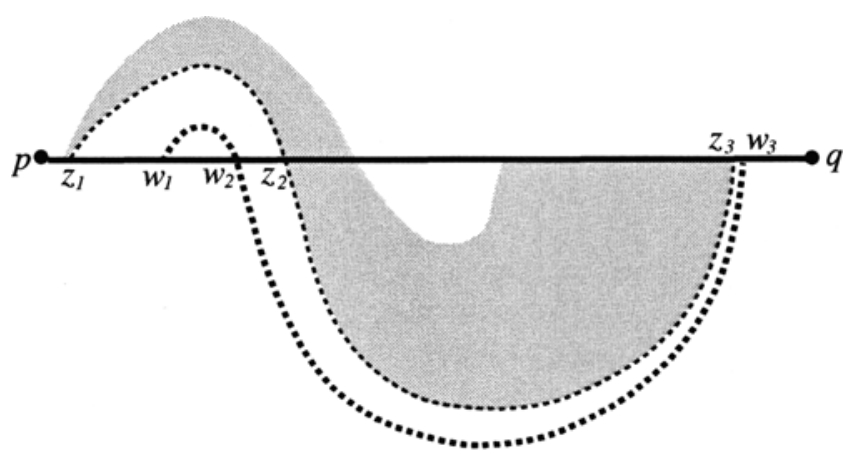

(i)

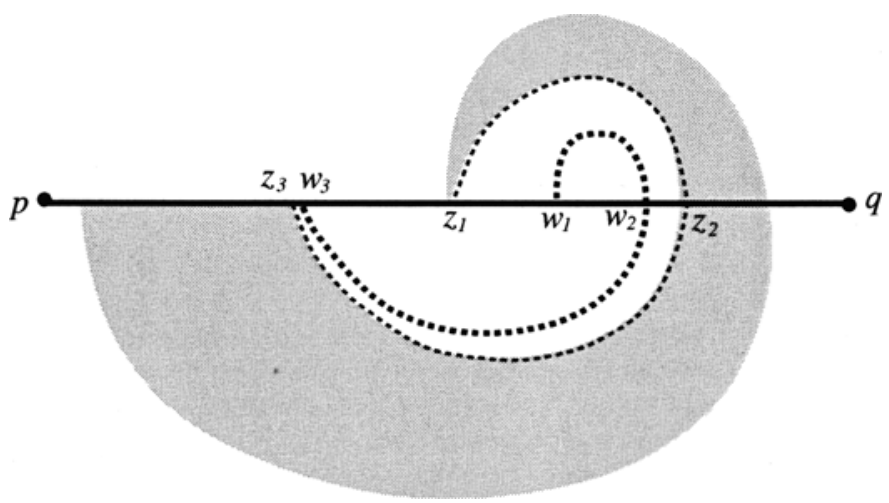

(ii)

Fig. A.3. The edge $p q$ intersects one boundary path of $\mathcal{H}_{z}$ at $z_{1}, z_{2}, z_{3}$ and one boundary path of $T$ at $w_{1}, w_{2}, w_{3}$. The figure shows two representative situations of the inverse horn $\mathcal{I}: z_{2}$ and $z_{3}$ are on the same side of $z_{1}$ in (i), but on different sides in (ii). Note that $w_{2}$ may be a point in $z_{1} w_{1}$ rather than $w_{1} z_{2}$, and $w_{3}$ of (ii) may lie in $z_{1} w_{1}$ rather than $z_{3} z_{1}$. 
For Fig. A.3(i), the maw widths of $\mathcal{H}_{z}$ at $z_{2}$ and at $z_{3}$ are both greater than $\left|z_{1} z_{2}\right|$ by Lemma A.1. So, the center path length of $\mathcal{I}$ from the midpoint of $w_{2} z_{2}$ to the midpoint of $w_{3} z_{3}$ is at least $2\left|z_{1} z_{2}\right|$. Since $\mathcal{H}_{z}$ does not self-intersect, Lemma A.2 implies that the maw width $I$ at $w_{3} z_{3}$ is at most $\left|w_{2} z_{2}\right|-\frac{4}{5}\left(2\left|z_{1} z_{2}\right|\right)<0$, as required. Besides Fig. A.3(i), there are three other situations to consider for $z_{2}$ and $z_{3}$ on the same side of $z_{1}$. First, $\mathcal{H}_{z}$ may leave $p q$ at $z_{1}$ and return to $p q$ at $z_{2}$ from below (rather than from above as shown in the figure). In doing so, the center path of $\mathcal{H}_{z}$ intersects some point $v$ on the line through $p q$ but not on $p q$. If $z_{2} \in p q \cap z_{1} v$, then the maw of $\mathcal{H}_{z}$ at $z_{2}$ contains $z_{1}$ (Lemma A.1) because the center path of $\mathcal{H}_{z}$ has traveled a distance larger than $\left|z_{1} v\right|$; if $z_{2} \notin p q \cap z_{1} v$, we can use the argument in the next paragraph. Second, $\mathcal{H}_{z}$ may leave $p q$ at $z_{2}$ and then return to $p q$ at $z_{3}$ from above, intersecting the line through $p q$ at points not on $p q$. In this case the above arguments also apply. Third, if $z_{3} \in z_{1} z_{2}$, then the maw of $\mathcal{H}_{z}$ again contains $z_{1}$.

For Fig. A.3(ii) with $z_{2}$ and $z_{3}$ on different sides of $z_{1}$, the center path point of $\mathcal{I}$ at $z_{1} w_{1}$ must be to the right of that at $z_{3} w_{3}$ since $z_{3} \notin z_{1} z_{2}$ and the maw width of $\mathcal{I}$ at $z_{3}$ is smaller than that at $z_{1}$. Thus, the center path of $\mathcal{I}$ traveled a distance of at least $l_{1}=\left|z_{1} w_{1}\right|$ to the midpoint of $z_{3} w_{3}$, and Lemma A.2 implies that its maw width $l_{3}=\left|z_{3} w_{3}\right|<l_{1}-\frac{4}{5} l_{1}=\frac{1}{5} l_{1}$. With this bound on $l_{3}$, a better bound on the distance traveled is $l_{1}+\left(l_{1} / 2-l_{3} / 2\right)>\frac{7}{5} l_{1}$, which in turn implies that $l_{3}$ is negative (Lemma A.2). Notice that the same argument can also handle the situation similar to Fig. A.3(ii) with $q \in w_{1} w_{2}$, i.e., $z_{2}$ and $w_{2}$ are not on $p q$.

Note that Lemma A.3 can be proved even if Lemma A.2 is weakened to having $l-l^{\prime}>\frac{67}{100} l_{c}$. This just requires a few more rounds of calculation of the bound on the distance traveled by the inverse horn for Fig. A.3(ii). This and because Lemma A.1 remains valid as long as $\beta>\pi / 3$, means we can pick $\alpha=\frac{11}{15} \pi$ (or $\beta=\frac{4}{15} \pi$ ) to derive $l-l^{\prime}>\frac{67}{100} l_{\mathrm{c}}$ for Lemma A.2 and, as a result, an improved angle bound.

\section{References}

1. I. Babuska and A. K. Aziz. On the angle condition in the finite element method. SIAM J. Numer. Anal. 13 (1976), 214-226.

2. R. E. Barnhill and F. F. Little. Three- and four-dimensional surfaces. Rocky Mountain J. Math. 14 (1984), 77-102.

3. M. Bern and D. Eppstein. Polynomial-size nonobtuse triangulation of polygons. Internat. J. Comput. Geom. Appl. 2(3) (1992), 241-255.

4. M. Bern and D. Eppstein. Mesh generation and optimal triangulation. In Computing in Euclidean Geom$e t r y$, 2nd edn., D. Z. Du and F. K. Hwang, eds., World Scientific, Singapore, 1995, pp. 47-123.

5. M. Bern, S. A. Mitchell, and J. Ruppert. Linear-size non-obtuse triangulation of polygons. Proc. 10th Ann. Symp. on Compulational Geometry, 1994, pp. 221-230.

6. J. D. Boissonnat. Shape reconstruction from planar cross sections. Comput. Vision Graphics Jmage Process. 44 (1988), 1-29.

7. J. Cavendish. Automatic triangulation of arbitrary planar domains for the finite element method. Internat. J. Numer. Methods Engrg. 8 (1974), 679-696.

8. T. K. Dey. Decompositions of Polyhedra in Three Dimensions. Technical Repor CSD-TR-91-056, Ph.D. Thesis, Computer Science Department, Purdue University, Indiana, 1991.

9. H. Edelsbrunner and N. Shah. Incremental topological flipping works for regular triangulations. Proc. 8th Ann. Symp. Computational Geometry, 1992, pp. 43-52. 
10. H. Edelsbrunner and T. S. Tan. An upper bound for conforming Delaunay triangulations. Discrete Comput. Geom. 10 (1993), 197-213.

11. H. Edelsbrunner, T. S. Tan, and R. Waupotitsch. An $O\left(n^{2} \log n\right)$ time algorithm for the minmax angle triangulation. SIAM J. Sci. Statist. Comput. 13 (1992), 994-1008.

12. J. A. Gregory. Error bounds for linear interpolation on triangles. In The Mathematics of Finite Element and Applications II, J. R. Whiteman, ed., Academic Press, New York, 1975, pp. 163-170.

13. L. J. Guibas and J. Stolf. Primitives for the manipulation of general subdivisions and the computation of Voronoi diagrams. ACM Trans. Graphics 4 (1985), 74-123.

14. C. L. Iawson. Software for $C^{l}$ surface interpolation. In Mathematical Software III, J. R. Rice, ed., Academic Press, New York, 1977, pp. 161-194.

15. E. A. Melissaratos and D. L. Souvaine. Coping with inconsistencies: a new approach to produce quality triangulations of polygonal domains with holes. Proc. 8th Ann. Symp. Computational Geometry, 1992. pp. $202-211$.

16. S. A. Mitchell. Mesh Generation with Provable Quality Bounds. Technical Report CS-TR-92-1327, Ph.D. Thesis, Computer Science Department, Comell University, New York, 1993.

17. S. A. Mitchell. Refining a triangulation of a planar straight-line graph to eliminate large angles. Proc. 34th Ann. IEEE Symp. on Foundations of Computer Science, 1993, pp. 583-591.

18. S. A. Mitchell. Finding a covering triangulation whose maximum angle is provably small. Austral. Comput. Sci. Comm. 16 (1994), 55-64.

19. L. Palios. Decomposition Problems in Computational Geometry. Technical Report CS-TR-368-92, Ph.D. Thesis, Computer Science Department, Princeton University, New Jersey, 1992.

20. J. Ruppert. A new and simple algorithm for quality two-dimensional mesh generation. Proc. 4th ACMSIAM Symp. on Discrete Algorithms, 1993, pp. 83-92.

21. T. S. Tan. Optimal Two-Dimensional Triangulations. Technical Report UIUCDCS-R-92-1783, Ph.D. Thesis, Computer Science Department, University of Illinois, Utbana, Illinois, 1993.

Received June 6, 1994, and in revised form February 5, 1995, and August 10, 1995. 